September 14, 2011

\title{
QUOTIENTS BY ACTIONS OF THE DERIVED GROUP OF A MAXIMAL UNIPOTENT SUBGROUP
}

\author{
DMITRI I. PANYUSHEV
}

\begin{abstract}
Let $U$ be a maximal unipotent subgroup of a connected semisimple group $G$ and $U^{\prime}$ the derived group of $U$. If $X$ is an affine $G$-variety, then the algebra of $U^{\prime}$-invariants, $k[X]^{U^{\prime}}$, is finitely generated and the quotient morphism $\pi: X \rightarrow X / / U^{\prime}=\operatorname{Spec} k[X]^{U^{\prime}}$ is well-defined. In this article, we study properties of such quotient morphisms, e.g. the property that all the fibres of $\pi$ are equidimensional. We also establish an analogue of the Hilbert-Mumford criterion for the null-cones with respect to $U^{\prime}$-invariants.
\end{abstract}

\section{INTRODUCTION}

The ground field $\mathbb{k}$ is algebraically closed and of characteristic zero. Let $G$ be a semisimple algebraic group with Lie algebra $\mathfrak{g}$. Fix a maximal unipotent subgroup $U \subset G$ and a maximal torus $T$ of the Borel subgroup $B=N_{G}(U)$. Set $U^{\prime}=(U, U)$. Let $X$ be an irreducible affine variety acted upon by $G$. The algebra of covariants (or, $U$-invariants) $\mathbb{k}[X]^{U}$ is a classical and important object in Invariant Theory. It is known that $\mathbb{k}[X]^{U}$ is finitely generated and has many other useful properties and applications, see e.g. [9, Ch. 3, §3]. For a factorial conical variety $X$ with rational singularities, there are interesting relations between the Poincaré series of the graded algebras $\mathbb{k}[X]$ and $\mathbb{k}[X]^{U}$, see $[3],[12, C h .5]$. Similar results for $U^{\prime}$-invariants are obtained in [14].

A surprising observation that stems from [14] is that, to a great extent, the theory of $U^{\prime}$ invariants is parallel to that of $U$-invariants. In this article, we elaborate on further aspects of this parallelism. Our main object is the quotient $\pi_{X, U^{\prime}}: X \rightarrow X / / U^{\prime}=\operatorname{Spec}\left(\mathbb{k}[X]^{U^{\prime}}\right)$. Specifically, we are interested in the property that $X / / U^{\prime}$ is an affine space and/or the morphism $\pi_{X, U^{\prime}}$ is equidimensional (i.e., all the fibres of $\pi_{X, U^{\prime}}$ have the same dimension). Our ultimate goal is to prove for $U^{\prime}$ an analogue of the Hilbert-Mumford criterion and to provide a classification of the irreducible representations $V$ of simple algebraic groups $G$ such that $\mathbb{k}[V]$ is a free $\mathbb{k}[V]^{U^{\prime}}$-module. We also develop some theory for $U^{\prime}$-actions on the affine prehomogeneous horospherical varieties of $G$ (S-varieties in terminology of [22]). As $U^{\prime}=\{1\}$ for $G=S L_{2}$, one sometimes has to assume that $G$ has no simple factors $S L_{2}$.

If $X$ has a $G$-fixed point, say $x_{0}$, then the fibre of $\pi_{X, U^{\prime}}$ containing $x_{0}$ is called the nullcone, and we denote it by $\mathfrak{N}_{U^{\prime}}(X)$. (The null-cone $\mathfrak{N}_{H}(X)$ can be defined for any subgroup

2010 Mathematics Subject Classification. 14L30, 17B20, 22 E46.

Key words and phrases. semisimple algebraic group, quotient, equidimensional morphism, invariant. 
$H \subset G$ such that $\mathbb{k}[X]^{H}$ is finitely generated.) If $G$ has no simple factors $S L_{2}$ nor $S L_{3}$, then the canonical affine model of $\mathbb{k}\left[G / U^{\prime}\right]$ constructed in [14, Sect. 2] consists of unstable points in the sense of GIT, and using this property we give a characterisation of $\mathfrak{N}_{U^{\prime}}(X)$ in terms of one-parameter subgroups of $T$. We call it the Hilbert-Mumford criterion for $U^{\prime}$. This is inspired by similar results of Brion for $U$-invariants [3, Sect. IV]. It is easily seen that $\mathfrak{N}_{U^{\prime}}(X) \subset \mathfrak{N}_{G}(X)$. Therefore $G \cdot \mathfrak{N}_{U^{\prime}}(X) \subset \mathfrak{N}_{G}(X)$. Using the Hilbert-Mumford criterion for $U^{\prime}$ we prove that $G \cdot \mathfrak{N}_{U^{\prime}}(X)=\mathfrak{N}_{G}(X)$ whenever $G$ has no simple factors $S L_{n}$. This should be compared with the result of Brion [3] that $G \cdot \mathfrak{N}_{U}(X)=\mathfrak{N}_{G}(X)$ for all $G$.

The $\mathcal{S}$-varieties are in one-to-one correspondence with the finitely generated monoids $\mathfrak{S}$ in the monoid $\mathfrak{X}_{+}$of dominant weights, and the $\mathcal{S}$-variety corresponding to $\mathfrak{S} \subset \mathfrak{X}_{+}$ is denoted by $\mathcal{C}(\mathfrak{S})$. We give exhaustive answers to three natural problems related to the actions of $U^{\prime}$ on $\mathcal{S}$-varieties. A set of fundamental weights $M$ is said to be sparse if the corresponding nodes of the Dynkin diagram are disjoint and, moreover, there does not exist any node (not in $M$ ) that is adjacent to two nodes from $M$. Our results are:

a) $\mathbb{k}[\mathcal{C}(\mathfrak{S})]^{U^{\prime}}$ is a polynomial algebra if and only if the monoid $\mathfrak{S}$ is generated by a set of fundamental weights;

b) $\mathbb{k}[\mathcal{C}(\mathfrak{S})]^{U^{\prime}}$ is a polynomial algebra and $\pi_{\mathcal{C}(\mathfrak{S}), U^{\prime}}$ is equidimensional if and only if the monoid $\mathfrak{S}$ is generated by a sparse set of fundamental weights;

c) the morphism $\pi_{\mathcal{C}(\mathfrak{S}), U^{\prime}}$ is equidimensional if and only if the convex polyhedral cone $\mathbb{R}^{+} \mathfrak{S}$ is generated by a sparse set of fundamental weights. (In particular, the cone $\mathbb{R}^{+} \mathfrak{S}$ is simplicial.)

Part a) is rather easy, while parts b) and c) require technical details related to the Bruhat decomposition of the flag variety associated with $\mathcal{C}(\mathfrak{S})$. If $\mathfrak{S}$ has one generator, say $\lambda$, and $\mathrm{R}(\lambda)$ is a simple $G$-module with highest weight $\lambda$, then $\mathcal{C}(\mathfrak{S})$ is the closure of the orbit of highest weight vectors in the dual $G$-module $\mathrm{R}(\lambda)^{*}$. Such a variety is denoted by $\mathcal{C}(\lambda)$. As in [22], we say that $\mathcal{C}(\lambda)$ is an HV-variety. Our results for HV-varieties are more complete. For instance, we compute the homological dimension of $\mathcal{C}(\lambda) / / U^{\prime}$ and prove that $\mathfrak{N}_{U^{\prime}}(\mathcal{C}(\lambda))$ is always of codimension 2 in $\mathcal{C}(\lambda)$. The criterion of part $b$ ) is then transformed into a sufficient condition applicable to a wider class of affine varieties:

Theorem 0.1. Suppose that $G$ acts on an irreducible affine variety $X$ such that (1) $\mathbb{k}[X]^{U}$ is a polynomial algebra and (2) the weights of free generators are fundamental, pairwise distinct, and form a sparse set. Then $\mathbb{k}[X]^{U^{\prime}}$ is also polynomial, of Krull dimension $2 \operatorname{dim} X / / U$, and the quotient $\pi_{X, U^{\prime}}: X \rightarrow X / / U^{\prime}$ is equidimensional.

This exploits the theory of "contractions of actions" of $G$ [15] and can be regarded as a continuation of our work in [13, Sect. 5], where the equidimensionality problem was considered for quotient morphism by $U$. For instance, under the hypotheses of Theorem 0.1, the morphism $\pi_{X, U}$ is also equidimensional. 
In [14], we obtained a classification of the irreducible representations of simple algebraic groups such that $\mathbb{k}[V]^{U^{\prime}}$ is a polynomial algebra. Now, using Theorem 0.1 and some ad hoc arguments, we extract from that list the representations having the additional property that $\pi_{V, U^{\prime}}$ is equidimensional. The resulting list is precisely the list of representations such that $\mathbb{k}[V]$ is a free $\mathbb{k}[V]^{U^{\prime}}$-module (such $G$-representations are said to be $U^{\prime}$-cofree).

This work is organized as follows. Section 1 contains auxiliary results on $\mathcal{S}$-varieties [22], $U^{\prime}$-invariants [14], and equidimensional morphisms. In Section 2, we consider $U^{\prime}$ actions on the HV-varieties. Section 3 is devoted to the $U^{\prime}$-actions on arbitrary $\mathcal{S}$-varieties. Here we prove results of items a) and b) above (Theorems 3.2, 3.4, and 3.7). In Section 4, we prove the general equidimensionality criterion for $\mathcal{S}$-varieties (item c)). The HilbertMumford criterion for $U^{\prime}$ and relations between two null-cones are discussed in Section 5. In Section 6, we prove Theorem 0.1 and obtain the classification of $U^{\prime}$-cofree representations of $G$.

Notation. If an algebraic group $Q$ acts regularly on an irreducible affine variety $X$, then $X$ is called a $Q$-variety and

- $Q_{x}=\{q \in Q \mid q \cdot x=x\}$ is the stabiliser of $x \in X$;

- $\mathbb{k}[X]^{Q}$ is the algebra of $Q$-invariant polynomial functions on $X$. If $\mathbb{k}[X]^{Q}$ is finitely generated, then $X / / Q:=\operatorname{Spec}\left(\mathbb{k}[X]^{Q}\right)$, and the quotient morphism $\pi_{Q}=\pi_{X, Q}: X \rightarrow X / / Q$ is the mapping associated with the embedding $\mathbb{k}[X]^{Q} \hookrightarrow \mathbb{k}[X]$.

Throughout, $G$ is a semisimple simply-connected algebraic group, $W=N_{G}(T) / T$ is the Weyl group, $B=T U$, and $r=\operatorname{rk} G$. Then

- $\Delta$ is the root system of $(G, T), \Pi=\left\{\alpha_{1}, \ldots, \alpha_{r}\right\} \subset \Delta$ are the simple roots corresponding to $U$, and $\varpi_{1}, \ldots, \varpi_{r}$ are the corresponding fundamental weights.

- The character group of $T$ is denoted by $\mathfrak{X}$. All roots and weights are regarded as elements of the $r$-dimensional real vector space $\mathfrak{X}_{\mathbb{R}}:=\mathfrak{X} \otimes \mathbb{R}$.

- ( , ) is a $W$-invariant symmetric non-degenerate bilinear form on $\mathfrak{X}_{\mathbb{R}}$ and $s_{i} \in W$ is the reflection corresponding to $\alpha_{i}$. For any $\lambda \in \mathfrak{X}_{+}$, let $\lambda^{*}$ denote the highest weight of the dual $G$-module, i.e., $\mathrm{R}(\lambda)^{*} \simeq \mathrm{R}\left(\lambda^{*}\right)$. The $\mu$-weight space of $\mathrm{R}(\lambda)$ is denoted by $\mathrm{R}(\lambda)_{\mu}$.

We refer to [21] for standard results on root systems and representations of semisimple algebraic groups.

\section{RECOLLECTIONS}

1.1. Horospherical varieties with a dense orbit. A $G$-variety $X$ is said to be horospherical if the stabiliser of any $x \in X$ contains a maximal unipotent subgroup of $G$. Following [22], affine horospherical varieties with a dense $G$-orbit are called $\mathcal{S}$-varieties. Let $\mathfrak{S}$ be a finitely generated monoid in $\mathfrak{X}_{+}$and $\left\{\lambda_{1}, \ldots, \lambda_{m}\right\}$ the minimal set of generators of $\mathfrak{S}$. Let 
$v_{-\lambda_{i}} \in \mathrm{R}\left(\lambda_{i}^{*}\right)$ be a lowest weight vector. Set $\boldsymbol{v}=\left(v_{-\lambda_{1}}, \ldots, v_{-\lambda_{m}}\right)$ and consider

$$
\mathcal{C}(\mathfrak{S}):=\overline{G \cdot v} \subset \mathrm{R}\left(\lambda_{1}^{*}\right) \oplus \cdots \oplus \mathrm{R}\left(\lambda_{m}^{*}\right) .
$$

Clearly, $\mathcal{C}(\mathfrak{S})$ is an $\mathcal{S}$-variety; conversely, each $\mathcal{S}$-variety is obtained in this way [22]. Write $\langle\mathfrak{S}\rangle$ for the linear span of $\mathfrak{S}$ in $\mathfrak{X}_{\mathbb{R}}$ and set rk $\mathfrak{S}=\operatorname{dim}_{\mathbb{R}}\langle\mathfrak{S}\rangle$. Let $L_{\mathfrak{S}}$ be the Levi subgroup such that $T \subset L_{\mathfrak{S}}$ and the roots of $L_{\mathfrak{S}}$ are those orthogonal to $\lambda_{1}, \ldots, \lambda_{m}$. Then $P_{\mathfrak{S}}=L_{\mathfrak{S}} N_{\mathfrak{S}}$ is the standard parabolic subgroup, with unipotent radical $N_{\mathfrak{S}} \subset U$.

Theorem 1.1 ([22]). The affine variety $\mathcal{C}(\mathfrak{S})$ has the following properties:

1. The algebra $\mathbb{k}[\mathcal{C}(\mathfrak{S})]$ is a multiplicity free $G$-module. More precisely, $\mathbb{k}[\mathcal{C}(\mathfrak{S})]=$ $\bigoplus_{\lambda \in \mathfrak{S}} \mathrm{R}(\lambda)$ and this decomposition is a multigrading, i.e., $\mathrm{R}(\lambda) \mathrm{R}(\mu)=\mathrm{R}(\lambda+\mu)$;

2. The $G$-orbits in $\mathcal{C}(\mathfrak{S})$ are in a one-to-one correspondence with the faces of the convex polyhedral cone in $\mathfrak{X}_{\mathbb{R}}$ generated by $\mathfrak{S}$;

3. $\mathcal{C}(\mathfrak{S})$ is normal if and only if $\mathbb{Z} \mathfrak{S} \cap \mathbb{Q}^{+} \mathfrak{S}=\mathfrak{S}$;

4. $\operatorname{dim} \mathcal{C}(\mathfrak{S})=\operatorname{dim} G / P_{\mathfrak{S}}+\mathrm{rk} \mathfrak{S}$.

If $\mathfrak{S}=\mathbb{N} \lambda$, then we write $\mathcal{C}(\lambda), P_{\lambda}, \ldots$ in place of $\mathcal{C}(\mathbb{N} \lambda), P_{\mathbb{N} \lambda}, \ldots$ The variety $\mathcal{C}(\lambda)$ is the closure of the $G$-orbit of highest weight vectors in $\mathrm{R}\left(\lambda^{*}\right)$. Such varieties are called HVvarieties; they are always normal. Recall that a $G$-variety $X$ is spherical, if $B$ has a dense orbit in $X$. Since $B \cdot \boldsymbol{v}$ is dense in $\mathcal{C}(\mathfrak{S})$, all $\mathcal{S}$-varieties are spherical. By [15, Theorem 10]), a normal spherical variety has rational singularities and therefore is Cohen-Macaulay. In particular, if $\mathfrak{S}$ is a free monoid, then $\mathcal{C}(\mathfrak{S})$ has rational singularities.

1.2. Generalities on $U^{\prime}$-invariants. We recall some results of [14] and thereby fix relevant notation. We regard $\mathfrak{X}$ as a poset with respect to the root order " $\preccurlyeq "$. This means that $\nu \preccurlyeq \mu$ if $\mu-\nu$ is a non-negative integral linear combination of simple roots. For any $\lambda \in \mathfrak{X}_{+}$, we fix a simple $G$-module $\mathrm{R}(\lambda)$ and write $\mathcal{P}(\lambda)$ for the set of $T$-weights of $\mathrm{R}(\lambda)$. Then $(\mathcal{P}(\lambda), \preccurlyeq)$ is a finite poset and $\lambda$ is its unique maximal element. Let $e_{i} \in \mathfrak{u}=\operatorname{Lie} U$ be a root vector corresponding to $\alpha_{i} \in \Pi$. Then $\left(e_{1}, \ldots, e_{r}\right)$ is a basis for Lie $\left(U / U^{\prime}\right)$.

The subspace of $U^{\prime}$-invariants in $\mathrm{R}(\lambda)$ has a nice description. Since $\mathrm{R}(\lambda)^{U^{\prime}}$ is acted upon by $B / U^{\prime}$, it is $T$-stable. Hence $\mathrm{R}(\lambda)^{U^{\prime}}=\bigoplus_{\mu \in \mathcal{J}_{\lambda}} \mathrm{R}(\lambda)_{\mu}^{U^{\prime}}$, where $\mathcal{J}_{\lambda}$ is a subset of $\mathcal{P}(\lambda)$.

Theorem 1.2 ([14, Theorem 1.6]). Suppose that $\lambda=\sum_{i=1}^{r} a_{i} \varpi_{i} \in \mathfrak{X}_{+}$. Then

(1) $\mathcal{J}_{\lambda}=\left\{\lambda-\sum_{i=1}^{r} b_{i} \alpha_{i} \mid 0 \leqslant b_{i} \leqslant a_{i} \forall i\right\}$;

(2) $\operatorname{dim} \mathrm{R}(\lambda)_{\mu}^{U^{\prime}}=1$ for all $\mu \in \mathcal{J}_{\lambda}$, i.e., $\mathrm{R}(\lambda)^{U^{\prime}}$ is a multiplicity free T-module;

(3) A nonzero $U^{\prime}$-invariant of weight $\lambda-\sum_{i=1}^{r} a_{i} \alpha_{i}$, say $f$, is a cyclic vector of the $U / U^{\prime}$ module $\mathrm{R}(\lambda)^{U^{\prime}}$. That is, the vectors $\left\{\left(\prod_{i=1}^{r} e_{i}^{b_{i}}\right)(\boldsymbol{f}) \mid 0 \leqslant b_{i} \leqslant a_{i} \forall i\right\}$ form a basis for $\mathrm{R}(\lambda)^{U^{\prime}}$. 
It follows from (1) and (2) that $\operatorname{dim} \mathrm{R}(\lambda)^{U^{\prime}}=\prod_{i=1}^{r}\left(a_{i}+1\right)$. In particular, $\operatorname{dim} \mathrm{R}\left(\varpi_{i}\right)^{U^{\prime}}=2$. The weight spaces $\mathrm{R}\left(\varpi_{i}\right)_{\varpi_{i}}$ and $\mathrm{R}\left(\varpi_{i}\right)_{\varpi_{i}-\alpha_{i}}$ are one-dimensional, and we fix corresponding nonzero weight vectors $f_{i}, \tilde{f}_{i}$ such that $e_{i}\left(\tilde{f}_{i}\right)=f_{i}$. That is, $\tilde{f}_{i}$ is a cyclic vector of $\mathrm{R}\left(\varpi_{i}\right)^{U^{\prime}}$.

The biggest $\mathcal{S}$-variety corresponds to the monoid $\mathfrak{S}=\mathfrak{X}_{+}$. Here

$$
\mathbb{k}[G / U]=\mathbb{k}\left[\mathcal{C}\left(\mathfrak{X}_{+}\right)\right]=\bigoplus_{\lambda \in \mathfrak{X}_{+}} \mathrm{R}(\lambda),
$$

and the multiplicative structure of $\mathbb{k}\left[\mathcal{C}\left(\mathfrak{X}_{+}\right)\right]$together with Theorem 1.2 imply

Theorem 1.3 (cf. [14, Theorem 1.8]). The algebra of $U^{\prime}$-invariants $\mathbb{k}\left[\mathcal{C}\left(\mathfrak{X}_{+}\right)\right]^{U^{\prime}}$ is freely generated by $f_{1}, \tilde{f}_{1}, \ldots, f_{r}, \tilde{f}_{r}$. Therefore, any basis for the $2 r$-dimensional vector space $\bigoplus_{i=1}^{r} \mathrm{R}\left(\varpi_{i}\right)^{U^{\prime}}$ yields a free generating system for $\mathbb{k}\left[\mathcal{C}\left(\mathfrak{X}_{+}\right)\right]^{U^{\prime}}$.

The algebra $\mathbb{k}[G / U]$ is sometimes called the flag algebra for $G$, because it can be realized as the multi-homogeneous coordinate ring of the flag variety $G / B$. More generally, we have

Theorem 1.4. If $\mathfrak{S}$ is generated by some fundamental weights, say $\left\{\varpi_{i} \mid i \in M\right\}$, then any basis for $\bigoplus_{i \in M} \mathrm{R}\left(\varpi_{i}\right)^{U^{\prime}}$ yields a free generating system for $\mathbb{k}[\mathcal{C}(\mathfrak{S})]^{U^{\prime}}$.

Proof. As in the proof of [14, Theorem 1.8], one observes that, for $\lambda=\sum_{i \in M} a_{i} \varpi_{i}$, the monomials $\left\{\prod_{i \in M} f_{i}^{b_{i}} \tilde{f}_{i}^{a_{i}-b_{i}} \mid 0 \leqslant b_{i} \leqslant a_{i}\right\}$ form a basis for the space $\mathrm{R}(\lambda)^{U^{\prime}}$. [Another way is to consider the natural embedding $\mathcal{C}(\mathfrak{S}) \hookrightarrow \mathcal{C}\left(\mathfrak{X}_{+}\right)$[22] and the surjective homomorphism $\mathbb{k}\left[\mathcal{C}\left(\mathfrak{X}_{+}\right)\right]^{U^{\prime}} \rightarrow \mathbb{k}[\mathcal{C}(\mathfrak{S})]^{U^{\prime}}$. $]$

Given $\lambda \in \mathfrak{X}_{+}$, we always consider a basis for $\mathrm{R}(\lambda)^{U^{\prime}}$ generated by a cyclic vector and elements $e_{i} \in \mathfrak{g}_{\alpha_{i}}$, i.e., a basis $\left\{f_{\mu} \in \mathrm{R}(\lambda)_{\mu} \mid \mu \in \mathcal{J}_{\lambda}\right\}$ such that

$$
e_{i}\left(f_{\mu}\right)= \begin{cases}f_{\mu+\alpha_{i}}, & \mu+\alpha_{i} \in \mathcal{J}_{\lambda}, \\ 0, & \mu+\alpha_{i} \notin \mathcal{J}_{\lambda} .\end{cases}
$$

However, for the fundamental $G$-modules $\mathrm{R}\left(\varpi_{i}\right)$, we write $f_{i}$ in place of $f_{\varpi_{i}}$ and $\tilde{f}_{i}$ in place of $f_{\varpi_{i}-\alpha_{i}}$.

1.3. Equidimensional morphisms and conical varieties. Let $\pi: X \rightarrow Y$ be a dominant morphism of irreducible algebraic varieties. We say that $\pi$ is equidimensional at $y \in Y$ if all irreducible components of $\pi^{-1}(y)$ are of dimension $\operatorname{dim} X-\operatorname{dim} Y$. Then $\pi$ is said to be equidimensional if it is equidimensional at any $y \in \pi(X)$. By a result of Chevalley [6, Ch. 5, n.5, Prop. 3], if $y=\pi(x)$ is a normal point, $\pi$ is equidimensional at $y$, and $\Omega \subset X$ is a neighbourhood of $x$, then $\pi(\Omega)$ is a neighbourhood of $y$. Consequently, an equidimensional morphism to a normal variety is open.

An affine variety $X$ is said to be conical if $\mathbb{k}[X]$ is $\mathbb{N}$-graded, $\mathbb{k}[X]=\bigoplus_{n \geqslant 0} \mathbb{k}[X]_{n}$, and $\mathbb{k}[X]_{0}=\mathbb{k}$. Then the point $x_{0}$ corresponding to the maximal ideal $\bigoplus_{n \geqslant 1} \mathbb{k}[X]_{n}$ is called the 
vertex. Geometrically, this means that $X$ is equipped with an action of the multiplicative group $\mathbb{k}^{\times}$such that $\left\{x_{0}\right\}$ is the only closed $\mathbb{k}^{\times}$-orbit in $X$.

Lemma 1.5. Suppose that both $X$ and $Y$ are conical, and $\pi: X \rightarrow Y$ is dominant and $\mathbb{k}^{\times}$equivariant. (Then $\pi\left(x_{0}\right)=: y_{0}$ is the vertex in $Y$.) If $Y$ is normal and $\pi$ is equidimensional at $y_{0}$, then $\pi$ is onto and equidimensional.

This readily follows from the above-mentioned result of Chevalley and standard inequalities for the dimension of fibres.

Remark 1.6. As $\mathfrak{S}$ lies in an open half-space of $\mathfrak{X}_{\mathbb{R}}$, taking a suitable $\mathbb{N}$-specialisation of the multi-grading of $\mathbb{k}[\mathcal{C}(\mathfrak{S})]$ shows that $\mathcal{C}(\mathfrak{S})$ is conical and the origin in $\mathrm{R}\left(\lambda_{1}^{*}\right) \oplus \cdots \oplus \mathrm{R}\left(\lambda_{m}^{*}\right)$ is its vertex. This implies that $\mathcal{C}(\mathfrak{S}) / / U^{\prime}$ is conical, too. We will apply the above lemma to the study of equidimensional quotient maps $\pi: \mathcal{C}(\mathfrak{S}) \rightarrow \mathcal{C}(\mathfrak{S}) / / U^{\prime}$. It is important that such $\pi$ appears to be onto.

The idea of applying Chevalley's result to the study of equidimensional quotients (by $U)$ is due to Vinberg and Gindikin [20].

\section{ACTIONS OF $U^{\prime}$ ON HV-VARIETIES}

Let $\mathcal{C}(\lambda)=\overline{G \cdot v_{-\lambda}} \subset \mathrm{R}\left(\lambda^{*}\right)$ be an HV-variety. The algebra $\mathbb{k}[\mathcal{C}(\lambda)]$ is $\mathbb{N}$-graded and its component of degree $n$ is $\mathrm{R}(n \lambda)$. Since $\mathcal{C}(\lambda)$ is normal, $\mathcal{C}(\lambda) / / U^{\prime}$ is normal, too.

Theorem 2.1. $\mathcal{C}(\lambda) / / U^{\prime}$ is an affine space if and only if $\lambda$ is a fundamental weight.

Proof. 1) Suppose that $\lambda$ is not fundamental, i.e., $\lambda=\cdots+a \varpi_{i}+b \varpi_{j}+\cdots$ with $a, b \geqslant 1$.

- If $i \neq j$, then $\mathrm{R}(\lambda)^{U^{\prime}}$ contains linearly independent vectors $f_{\lambda}, f_{\lambda-\alpha_{i}}, f_{\lambda-\alpha_{j}}, f_{\lambda-\alpha_{i}-\alpha_{j}}$ that occur in any minimal generating system, since $\mathbb{k}[\mathcal{C}(\lambda)]_{1} \simeq \mathrm{R}(\lambda)$. Using the relations $e_{i}\left(f_{\lambda-\alpha_{i}-\alpha_{j}}\right)=f_{\lambda-\alpha_{j}}$, etc., one easily verifies that

$$
p=f_{\lambda} f_{\lambda-\alpha_{i}-\alpha_{j}}-f_{\lambda-\alpha_{i}} f_{\lambda-\alpha_{j}}
$$

is a $U$-invariant function on $\mathcal{C}(\lambda)$, of degree 2 . The only highest weight in degree 2 is $2 \lambda$. Since the weight of $p$ is not $2 \lambda$, we must have $p \equiv 0$, and this is a non-trivial relation.

- If $i=j$, then the coefficient of $\varpi_{i}$ is at least 2 and we consider vectors $f_{\lambda}, f_{\lambda-\alpha_{i}}$, $f_{\lambda-2 \alpha_{i}} \in \mathrm{R}(\lambda)^{U^{\prime}}$. Then $\tilde{p}=2 f_{\lambda} f_{\lambda-2 \alpha_{i}}-f_{\lambda-\alpha_{i}}^{2}$ is a $U$-invariant function of degree 2 and weight $2\left(\lambda-\alpha_{i}\right)$, and this yields the relation $\tilde{p}=0$ in $\mathbb{k}[\mathcal{C}(\lambda)]^{U^{\prime}}$.

2) If $\lambda=\varpi_{i}$, then $\operatorname{dim} \mathrm{R}\left(\varpi_{i}\right)^{U^{\prime}}=2$ and $\mathcal{C}\left(\varpi_{i}\right) / / U^{\prime} \simeq \mathbb{A}^{2}$ by Theorem 1.4.

For an affine variety $X$, let edim $X$ denote the minimal number of generators of $\mathbb{k}[X]$ and $\mathrm{hd}(X)$ the homological dimension of $\mathbb{k}[X]$. If $\mathbb{k}[X]$ is a graded Cohen-Macaulay algebra, then $\operatorname{hd}(X)=\operatorname{edim} X-\operatorname{dim} X[17$, Ch. IV] 
Theorem 2.2. If $\lambda=\sum_{i=1}^{r} a_{i} \varpi_{i} \in \mathfrak{X}_{+}$, then

(i) $\operatorname{dim} \mathcal{C}(\lambda) / / U^{\prime}=1+\#\left\{j \mid a_{j} \neq 0\right\}$;

(ii) the graded algebra $\mathbb{k}[\mathcal{C}(\lambda)]^{U^{\prime}}$ is generated by functions of degree one, i.e., by the space $\mathrm{R}(\lambda)^{U^{\prime}}$, and $\operatorname{edim} \mathcal{C}(\lambda) / / U^{\prime}=\prod_{i=1}^{r}\left(a_{i}+1\right)$.

Proof. (i) Recall that $P_{\lambda}=L_{\lambda} N_{\lambda}$ is the standard parabolic subgroup associated with $\mathcal{C}(\lambda)$ and the simple roots of $L_{\lambda}$ are those orthogonal to $\lambda$. Set $k=\#\left\{j \mid a_{j} \neq 0\right\}$. Then $\operatorname{srk} L_{\lambda}:=\operatorname{rk}\left(L_{\lambda}, L_{\lambda}\right)=\operatorname{rk} G-k$ and $\operatorname{dim} \mathcal{C}(\lambda)=\operatorname{dim} N_{\lambda}+1$. Since $U \cdot\left(\mathbb{k} v_{-\lambda}\right)$ is dense in $\mathcal{C}(\lambda), U\left(L_{\lambda}\right):=U \cap L_{\lambda}$ is a generic stabiliser for the $U$-action on $\mathcal{C}(\lambda)$. By [14, Lemma 2.5], the minimal dimension of stabilisers for the $U^{\prime}$-action on $\mathcal{C}(\lambda)$ equals $\operatorname{dim}\left(U\left(L_{\lambda}\right) \cap U^{\prime}\right)=$ $\operatorname{dim} U\left(L_{\lambda}\right)-\operatorname{srk} L_{\lambda}$. Consequently,

$$
\begin{aligned}
& \operatorname{dim} \mathcal{C}(\lambda) / / U^{\prime}=\operatorname{dim} \mathcal{C}(\lambda)-\operatorname{dim} U^{\prime}+\min _{x \in \mathcal{C}(\lambda)} \operatorname{dim} U_{x}^{\prime}= \\
& \quad=\operatorname{dim} N_{\lambda}+1-(\operatorname{dim} U-\operatorname{rk} G)+\left(\operatorname{dim} U\left(L_{\lambda}\right)-\operatorname{srk} L_{\lambda}\right)=1+\operatorname{rk} G-\operatorname{srk} L_{\lambda}=1+k .
\end{aligned}
$$

(ii) By Theorem 1.2, $\operatorname{dim} \mathrm{R}(\lambda)^{U^{\prime}}=\prod_{i=1}^{r}\left(a_{i}+1\right)$, which shows that $\operatorname{edim} \mathcal{C}(\lambda) / / U^{\prime} \geqslant$ $\prod_{i=1}^{r}\left(a_{i}+1\right)$. Therefore, it suffices to prove that the graded algebra $\mathbb{k}[\mathcal{C}(\lambda)]^{U^{\prime}}$ is generated by elements of degree 1 . The weights of $U^{\prime}$-invariants of degree $n$ are

$$
\mathcal{J}_{n \lambda}=\left\{n \lambda-\sum_{i} b_{i} \alpha_{i} \mid b_{i}=0,1, \ldots, n a_{i}\right\} .
$$

In particular,

$$
\mathcal{J}_{\lambda}=\left\{\lambda-\sum_{i} b_{i} \alpha_{i} \mid b_{i}=0,1, \ldots, a_{i}\right\} .
$$

Obviously, each element of $\mathcal{J}_{n \lambda}$ is a sum of $n$ elements of $\mathcal{J}_{\lambda}$. Since $\mathrm{R}(n \lambda)^{U^{\prime}}$ is a multiplicity free $T$-module, this space is spanned by products of $n$ elements of $\mathrm{R}(\lambda)^{U^{\prime}}$.

Corollary 2.3. We have hd $\left(\mathcal{C}(\lambda) / / U^{\prime}\right)=\prod_{i=1}^{r}\left(1+a_{i}\right)-1-\#\left\{j \mid a_{j} \neq 0\right\}$. Therefore,

- $\operatorname{hd}\left(\mathcal{C}(\lambda) / / U^{\prime}\right)=0$ if and only if $\lambda$ is fundamental;

- $\operatorname{hd}\left(\mathcal{C}(\lambda) / / U^{\prime}\right)=1$ if and only if $\lambda=\varpi_{i}+\varpi_{j}$ or $2 \varpi_{i}$.

Proof. As it was mentioned above, the HV-varieties have rational singularities. In view of [14, Theorem 2.3], $\mathcal{C}(\lambda) / / U^{\prime}$ also has rational singularities and in particular is CohenMacaulay. Hence $\operatorname{hd}\left(\mathcal{C}(\lambda) / / U^{\prime}\right)=\operatorname{edim} \mathcal{C}(\lambda) / / U^{\prime}-\operatorname{dim} \mathcal{C}(\lambda) / / U^{\prime}$.

Remark 2.4. 1) As above, $k=\operatorname{rk} G-\operatorname{srk} L_{\lambda}$ and hence $\operatorname{dim} \mathcal{C}(\lambda) / / U^{\prime}=k+1$. Another consequence of Theorems 1.2 and 2.2 is that $\mathcal{C}(\lambda) / / U^{\prime}$ is a toric variety with respect to $\mathbb{k}^{\times} \times T$, where $\mathbb{k}^{\times}$acts on $\mathrm{R}\left(\lambda^{*}\right)$ (and hence on $\mathcal{C}(\lambda)$ ) by homotheties. Note that the $T$ action on $\mathcal{C}(\lambda) / / U^{\prime}$ has a non-effectivity kernel of dimension $\mathrm{rk} G-k$. The quotient morphism $\pi_{\mathcal{C}(\lambda), U^{\prime}}$ has the following description. Let $\operatorname{ann}\left(\mathrm{R}(\lambda)^{U^{\prime}}\right)$ be the annihilator of $\mathrm{R}(\lambda)^{U^{\prime}}$ in $\mathrm{R}\left(\lambda^{*}\right)$. Then $\left(\mathrm{R}(\lambda)^{U^{\prime}}\right)^{*}=\mathrm{R}\left(\lambda^{*}\right) / \operatorname{ann}\left(\mathrm{R}(\lambda)^{U^{\prime}}\right)$ and $\pi_{\mathcal{C}(\lambda), U^{\prime}}$ is the restriction to $\mathcal{C}(\lambda)$ of the 
projection $\mathrm{R}\left(\lambda^{*}\right) \rightarrow\left(\mathrm{R}(\lambda)^{U^{\prime}}\right)^{*}$. Thus, $\mathcal{C}(\lambda) / / U^{\prime}$ is embedded in the vector space $\left(\mathrm{R}(\lambda)^{U^{\prime}}\right)^{*}$. Consequently, $\mathbb{P}\left(\mathcal{C}(\lambda) / / U^{\prime}\right) \subset \mathbb{P}\left(\left(\mathrm{R}(\lambda)^{U^{\prime}}\right)^{*}\right)$ is a normal toric variety with respect to $T$. As is well-known, a projective toric $T$-variety can be described via a convex polytope in $\mathfrak{X}_{\mathbb{Q}}[7,5.8]$. The polytope corresponding to $\mathbb{P}\left(\mathcal{C}(\lambda) / / U^{\prime}\right)$ is the convex hull of $\mathcal{J}_{\lambda}$. It is a $k$-dimensional parallelepiped, in particular, a simple polytope. It follows that the corresponding complete fan is simplicial. Therefore the complex cohomology of $\mathbb{P}\left(\mathcal{C}(\lambda) / / U^{\prime}\right)$ satisfies Poincaré duality and has a number of other good properties, see [7, $§ 14]$.

2) Along with the toric structure (i.e., a dense $T$-orbit), the projective variety $\mathbb{P}\left(\mathcal{C}(\lambda) / / U^{\prime}\right)$ also has a dense orbit of the commutative unipotent group $U / U^{\prime}$.

\section{ACTIONS OF $U^{\prime}$ ON ARBITRARY $\mathcal{S}$-VARIETIES}

Let $\mathcal{C}(\mathfrak{S})$ be an $\mathcal{S}$-variety. In this section, we answer the following questions:

- When is $\mathcal{C}(\mathfrak{S}) / / U^{\prime}$ an affine space?

- Suppose that $\mathcal{C}(\mathfrak{S}) / / U^{\prime}$ is an affine space. When is $\pi_{\mathcal{C}(\mathfrak{S}), U^{\prime}}$ equidimensional?

We begin with a formula for $\operatorname{dim} \mathcal{C}(\mathfrak{S}) / / U^{\prime}$, which generalises Theorem 2.2(i).

Proposition 3.1. $\operatorname{dim} \mathcal{C}(\mathfrak{S}) / / U^{\prime}=\operatorname{rk} \mathfrak{S}+\left(\operatorname{rk} G-\operatorname{srk} L_{\mathfrak{S}}\right)$.

Proof. By Theorem 1.1, $\operatorname{dim} \mathcal{C}(\mathfrak{S})=\operatorname{dim} N_{\mathfrak{S}}+\mathrm{rk} \mathfrak{S}$ and $\operatorname{dim} \mathcal{C}(\mathfrak{S}) / / U=$ rk $\mathfrak{S}$. This readily implies that $U\left(L_{\mathfrak{S}}\right):=U \cap L_{\mathfrak{S}}$ is a generic stabiliser for the $U$-action on $\mathcal{C}(\mathfrak{S})$. By [14, Lemma 2.5], the minimal dimension of stabilisers for the $U^{\prime}$-action on $\mathcal{C}(\mathfrak{S})$ equals $\operatorname{dim}\left(U\left(L_{\mathfrak{S}}\right) \cap U^{\prime}\right)=\operatorname{dim} U\left(L_{\mathfrak{S}}\right)-\operatorname{srk} L_{\mathfrak{S}}$. Consequently,

$$
\begin{aligned}
& \operatorname{dim} \mathcal{C}(\mathfrak{S}) / / U^{\prime}=\operatorname{dim} \mathcal{C}(\mathfrak{S})-\operatorname{dim} U^{\prime}+\min _{x \in \mathcal{C}(\mathfrak{S})} \operatorname{dim} U_{x}^{\prime}= \\
& =\operatorname{dim} N_{\mathfrak{S}}+\text { rk } \mathfrak{S}-(\operatorname{dim} U-\mathrm{rk} G)+\left(\operatorname{dim} U\left(L_{\mathfrak{S}}\right)-\operatorname{srk} L_{\mathfrak{S}}\right)=\operatorname{rk} \mathfrak{S}+\left(\mathrm{rk} G-\operatorname{srk} L_{\mathfrak{S}}\right) .
\end{aligned}
$$

Here we use the fact that $U$ is a semi-direct product of $N_{\mathfrak{S}}$ and $U\left(L_{\mathfrak{S}}\right)$.

Remark. Note that rk $\mathfrak{S} \leqslant \mathrm{rk} G-\operatorname{srk} L_{\mathfrak{S}}$, and the equality here is equivalent to the fact that the space $\langle\mathfrak{S}\rangle$ has a basis that consists of fundamental weights.

Theorem 3.2. Let $\mathfrak{S} \subset \mathfrak{X}_{+}$be an arbitrary finitely generated monoid. Then $\mathcal{C}(\mathfrak{S}) / / U^{\prime}$ is an affine space if and only if $\mathfrak{S}$ is generated by fundamental weights.

Proof. 1) Suppose that $\mathcal{C}(\mathfrak{S}) / / U^{\prime}$ is an affine space. If $\lambda$ is a generator of $\mathfrak{S}$, then any generating system of $\mathbb{k}[\mathcal{C}(\mathfrak{S})]^{U^{\prime}}$ contains a basis for $\mathrm{R}(\lambda)^{U^{\prime}}$. Arguing as in the proof of Theorem 2.1, we conclude that $\lambda$ must be a fundamental weight. [Another way is to use Proposition 3.1 and the inequality $\operatorname{dim} \mathcal{C}(\mathfrak{S}) / / U^{\prime} \geqslant 2$ rk S .]

2) The converse is contained in Theorem 1.4. 
In the rest of this section, we only consider monoids generated by fundamental weights. Fix a numbering of the simple roots (fundamental weights). For any $M \subset\{1,2, \ldots, r\}$, let $\mathcal{C}(M)$ denote the $\mathcal{S}$-variety corresponding to the monoid $\mathfrak{S}=\sum_{i \in M} \mathbb{N} \varpi_{i}$. Our aim is to characterise the subsets $M$ having the property that $\pi_{U^{\prime}}: \mathcal{C}(M) \rightarrow \mathcal{C}(M) / / U^{\prime}$ is equidimensional. The origin (vertex) is the only $G$-fixed point of $\mathcal{C}(M)$ and the corresponding fibre of $\pi_{U^{\prime}}$ (the null-cone) is denoted by $\mathfrak{N}_{U^{\prime}}(M)$.

Recall that $\mathbb{k}[\mathcal{C}(M)]$ is a graded Cohen-Macaulay ring and $\mathbb{k}[\mathcal{C}(M)]^{U^{\prime}}$ is a polynomial algebra freely generated by $\left\{f_{i}, \tilde{f}_{i} \mid i \in M\right\}$ (Theorem 1.4). Therefore, $\pi_{U^{\prime}}$ is equidimensional if and only if the functions $\left\{f_{i}, \tilde{f}_{i} \mid i \in M\right\}$ form a regular sequence in $\mathbb{k}[\mathcal{C}(M)]$ if and only if $\operatorname{dim} \mathfrak{N}_{U^{\prime}}(M)=\operatorname{dim} \mathcal{C}(M)-2(\# M)[16, \S 17]$.

Definition 1. A subset $M \subset\{1, \ldots, r\}$ is said to be sparse, if 1) the roots $\alpha_{i}$ with $i \in M$ are pairwise orthogonal, i.e., disjoint in the Dynkin diagram; 2) there are no $i, j \in M$ and no $k \notin M$ such that $\left(\alpha_{k}, \alpha_{i}\right)<0$ and $\left(\alpha_{k}, \alpha_{j}\right)<0$, i.e., $\alpha_{k}$ is adjacent to both $\alpha_{i}$ and $\alpha_{j}$. Accordingly, we say that a certain set of fundamental weights (simple roots) is sparse.

Clearly, if $M$ is sparse and $J \subset M$, then $J$ is also sparse.

Lemma 3.3. Let $\alpha_{i_{1}}, \ldots, \alpha_{i_{l}}$ be a sequence of different simple roots such that $\alpha_{i_{j}}, \alpha_{i_{j+1}}$ are adjacent for $j=1,2, \ldots, l-1)$. Then $\mu:=\varpi_{i_{1}}-\sum_{j=1}^{l} \alpha_{i_{j}}$ is a weight of $\mathrm{R}\left(\varpi_{i_{1}}\right)$ and $\operatorname{dim} \mathrm{R}\left(\varpi_{i_{1}}\right)_{\mu}=1$.

Proof. The first assertion is easily proved by induction on $l$. The second assertion follows from [1, Prop. 2.2]

Theorem 3.4. If the quotient $\pi_{U^{\prime}}: \mathcal{C}(M) \rightarrow \mathcal{C}(M) / / U^{\prime}$ is equidimensional, then $M$ is sparse.

Proof. As we already know, $\mathbb{k}[\mathcal{C}(M)]^{U^{\prime}}$ is freely generated by the functions $\left\{f_{i}, \tilde{f}_{i} \mid i \in M\right\}$. Assuming that $M$ is not sparse, we point out certain relations in $\mathbb{k}[\mathcal{C}(M)]$, which show that these free generators do not form a regular sequence. There are two possibilities for that.

- Suppose first that $\alpha_{i}$ and $\alpha_{j}$ are adjacent simple roots for some $i, j \in M$. Then $\lambda_{i j}:=\varpi_{i}+\varpi_{j}-\alpha_{i}-\alpha_{j}$ is dominant. Consider upper parts of the Hasse diagrams of weight posets for $\mathrm{R}\left(\varpi_{i}\right)$ and $\mathrm{R}\left(\varpi_{j}\right)$ :

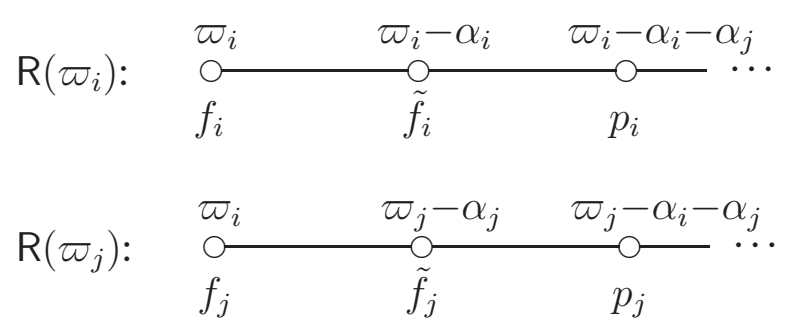

In these figures, each node depicts a weight space, and we put the weight over the node and a weight vector under the node. There can be other edges incident to the node $\varpi_{i}-\alpha_{i}$ 
(if there exist other simple roots adjacent to $\alpha_{i}$ ), but we do not need them. By Lemma 3.3, the weight spaces $\mathrm{R}\left(\varpi_{i}\right)_{\varpi_{i}}, \mathrm{R}\left(\varpi_{i}\right)_{\varpi_{i}-\alpha_{i}}$, and $\mathrm{R}\left(\varpi_{i}\right)_{\varpi_{i}-\alpha_{i}-\alpha_{j}}$ are one-dimensional. Here $f_{i}, \tilde{f}_{i}$, and $p_{i}$ are normalised such that $e_{i}\left(\tilde{f}_{i}\right)=f_{i}$ and $e_{j}\left(p_{i}\right)=\tilde{f}_{i}$; and likewise for $\mathrm{R}\left(\varpi_{j}\right)$. Note also that $e_{i}\left(p_{i}\right)=0$, since $\varpi_{i}-\alpha_{j}$ is not a weight of $\mathrm{R}\left(\varpi_{i}\right)$. It is then easily seen that

$$
f_{i} \otimes p_{j}-\tilde{f}_{i} \otimes \tilde{f}_{j}+p_{i} \otimes f_{j}
$$

is a $U$-invariant of weight $\lambda_{i j}$ in $\mathrm{R}\left(\varpi_{i}\right) \otimes \mathrm{R}\left(\varpi_{j}\right)$. However, only the Cartan component of $\mathrm{R}\left(\varpi_{i}\right) \otimes \mathrm{R}\left(\varpi_{j}\right)$ survives in the algebra $\mathbb{k}[\mathcal{C}(M)]$, i.e., in the product $\mathrm{R}\left(\varpi_{i}\right) \cdot \mathrm{R}\left(\varpi_{j}\right)$. Consequently, $f_{i} p_{j}-\tilde{f}_{i} \tilde{f}_{j}+p_{i} f_{j}=0$ in $\mathbb{k}[\mathcal{C}(M)]$. This means that $\left(f_{i}, f_{j}, \tilde{f}_{i}, \tilde{f}_{j}\right)$ is not a regular sequence in $\mathbb{k}[\mathcal{C}(M)]$.

- Yet another possibility is that there are $k \notin M$ and $i, j \in M$ such that $\alpha_{k}$ is adjacent to both $\alpha_{i}$ and $\alpha_{j}$. Here one verifies that $\tilde{\lambda}_{i j}:=\varpi_{i}+\varpi_{j}-\alpha_{i}-\alpha_{k}-\alpha_{j}$ is dominant. In this situation, we need larger fragments of the weight posets:

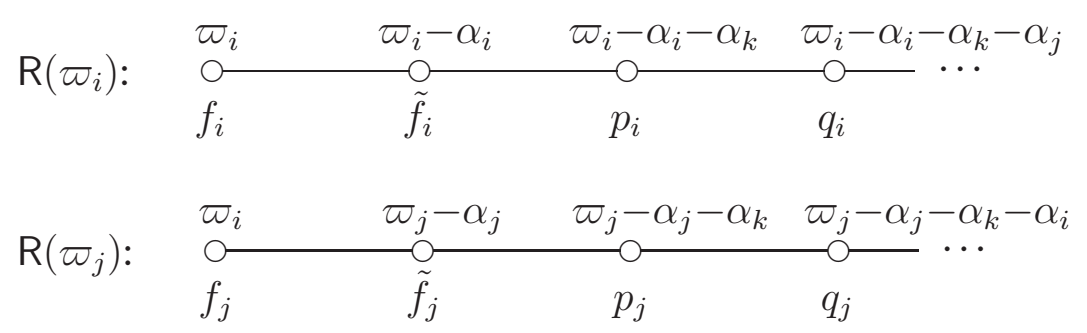

Here all the weight spaces are one-dimensional by Lemma 3.3, and we follow the same conventions as above. Additionally, we assume that $e_{j}\left(q_{i}\right)=p_{i}$. Note that $e_{k}\left(q_{i}\right)=0$ and $e_{i}\left(q_{i}\right)=0$, since neither $\varpi_{i}-\alpha_{i}-\alpha_{j}$ nor $\varpi_{i}-\alpha_{k}-\alpha_{j}$ is a weight of $\mathrm{R}\left(\varpi_{i}\right)$. (And likewise for $\mathrm{R}\left(\varpi_{j}\right)$.) Then $f_{i} \otimes q_{j}-\tilde{f}_{i} \otimes p_{j}+p_{i} \otimes \tilde{f}_{j}-q_{i} \otimes f_{j}$ is a $U$-invariant of weight $\tilde{\lambda}_{i j}$, and hence

$$
f_{i} q_{j}-\tilde{f}_{i} p_{j}+p_{i} \tilde{f}_{j}-q_{i} f_{j}=0
$$

in $\mathbb{k}[\mathcal{C}(M)]$ for the same reason as above. This again implies that $\left(f_{i}, f_{j}, \tilde{f}_{i}, \tilde{f}_{j}\right)$ is not a regular sequence in $\mathbb{k}[\mathcal{C}(M)]$.

Example 3.5. Let $\mathfrak{g}=\mathfrak{s l}_{4}$ and $M=\{1,3\}$ in the usual numbering of $\Pi$. Then $\operatorname{dim} \mathrm{R}\left(\varpi_{1}\right)=$ $\operatorname{dim} \mathrm{R}\left(\varpi_{3}\right)=4$ and $\operatorname{dim} \mathcal{C}(M)=7$. In this case, the above 4-node fragments provide the whole weight posets. Therefore, $\mathrm{R}\left(\varpi_{1}\right)=\left\langle f_{1}, \tilde{f}_{1}, p_{1}, q_{1}\right\rangle, \mathrm{R}\left(\varpi_{3}\right)=\left\langle f_{3}, \tilde{f}_{3}, p_{3}, q_{3}\right\rangle$, and (3.1) with $(i, j)=(1,3)$ is the equation of the hypersurface $\mathcal{C}(M)$. Since $\operatorname{dim} \mathcal{C}(M) / / U^{\prime}=4$ and $\mathfrak{N}_{U^{\prime}}(M) \supset\left\langle p_{1}, q_{1}, p_{3}, q_{3}\right\rangle$, the morphism $\pi_{U^{\prime}}$ is not equidimensional.

To prove the converse to Theorem 3.4, we need some preparations. Recall that the partial order " $\preccurlyeq "$ is defined in 1.2. We also write $\nu \prec \mu$ if $\nu \preccurlyeq \mu$ and $\mu \neq \nu$.

Lemma 3.6. Suppose that $M$ is sparse and $w \in W$ has the property that $w\left(\varpi_{i}\right) \prec \varpi_{i}-\alpha_{i}$ for all $i \in M$. Then $\ell(w) \geqslant 2 \cdot \#(M)$. 
Proof. Since $w\left(\varpi_{i}\right) \prec \varpi_{i}$, any reduced decomposition of $w$ contains $s_{i}$. Furthermore, since $w\left(\varpi_{i}\right) \prec \varpi_{i}-\alpha_{i}$, there exists a node $i^{\prime}$ adjacent to $i$ such that $w\left(\varpi_{i}\right) \preccurlyeq \varpi_{i}-\alpha_{i}-\alpha_{i^{\prime}}$. Therefore, $w$ must also contain the reflection $s_{i^{\prime}}$. Because $M$ is sparse, all the reflections $\left\{s_{i}, s_{i^{\prime}} \mid i \in M\right\}$ are different. Thus, $\ell(w) \geqslant 2 \cdot \#(M)$.

For any $I \subset \Pi$, we consider the following objects. Let $P_{I}=L_{I} N_{I}$ be the standard parabolic subgroup of $G$. Here $L_{I}$ is the Levi subgroup whose set of simple roots is $I$ and $N_{I}$ is the unipotent radical of $P_{I}$. Then $P_{I}^{-}=L_{I} N_{I}^{-}$is the opposite parabolic subgroup of $G$. We also need the factorisation

$$
W=W^{I} \times W_{I},
$$

where $W_{I}$ is the subgroup generated by $\left\{s_{i} \mid \alpha_{i} \in I\right\}$ and $W^{I}$ is the set of representatives of minimal length for $W / W_{I}[8,1.10]$. It is also true that $W^{I}=\left\{w \in W \mid w\left(\alpha_{i}\right) \in \Delta^{+} \forall \alpha_{i} \in I\right\}$ $[8,5.4]$. If $I=\{\alpha \in \Pi \mid(\alpha, \lambda)=0\}$ for some $\lambda \in \mathfrak{X}_{+}$, then we write $P_{\lambda}, W_{\lambda}, W^{\lambda}$, etc.

For each $w \in W$, we fix a representative, $\dot{w}$, in $N_{G}(T)$. As is well-known, the $U$-orbits in $G / P_{I}^{-}$can be parametrised by $W^{I}$, and letting $\mathcal{O}(w)=U \dot{w} P_{I}^{-} \subset G / P_{I}^{-}\left(w \in W^{I}\right)$, we have $G / P_{I}^{-}=\sqcup_{w \in W^{I}} \mathcal{O}(w)$ and $\operatorname{codim} \mathcal{O}(w)=\ell(w)$.

Theorem 3.7. If $M \subset\{1, \ldots, r\}$ is sparse, then the quotient $\pi_{U^{\prime}}: \mathcal{C}(M) \rightarrow \mathcal{C}(M) / / U^{\prime}$ is equidimensional.

Proof. Set $m=\# M$ and $I=\Pi \backslash\left\{\alpha_{i} \mid i \in M\right\}$. Consider $\boldsymbol{v}=\sum_{i \in M} v_{-\varpi_{i}} \in \bigoplus_{i \in M} \mathrm{R}\left(\varpi_{i}^{*}\right)$. As explained in Subsection 1.1, then $\mathcal{C}(M) \simeq \overline{G \cdot \boldsymbol{v}}$ and $\operatorname{dim} \mathcal{C}(M)=\operatorname{dim} G / P_{I}^{-}+m$. We also have $\operatorname{dim} \mathcal{C}(M) / / U^{\prime}=2 m$. Therefore, our goal is to prove that $\operatorname{dim} \mathfrak{N}_{U^{\prime}}(M) \leqslant \operatorname{dim} G / P_{I}^{-}-$ $m$.

Set $V=\overline{T \cdot \boldsymbol{v}}=\bigoplus_{i \in M} \mathbb{k} v_{-\varpi_{i}}$. It is an $m$-dimensional subspace of $\bigoplus_{i \in M} \mathrm{R}\left(\varpi_{i}^{*}\right)$, which is contained in $\mathcal{C}(M)$ and is $P_{I}^{-}$-stable. Recall that $G \times \times_{P_{I}^{-}} V$ is a homogeneous vector bundle on $G / P_{I}^{-}$. A typical element of it is denoted by $g * v$, where $g \in G$ and $v=\sum_{i \in M} v_{i} \in V$. Our main tool for estimating $\operatorname{dim} \mathfrak{N}_{U^{\prime}}(M)$ is the following diagram:

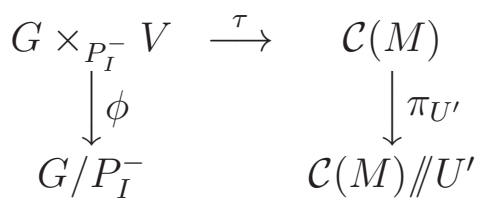

where $\phi(g * v):=g P_{I}^{-}$and $\tau(g * v):=g \cdot v$. Note that $\mathfrak{N}_{U^{\prime}}(M)$ is $B$-stable, and hence so is $\tau^{-1}\left(\mathfrak{N}_{U^{\prime}}(M)\right)$. It is easily seen that the morphism $\tau$ is birational and therefore it is an equivariant resolution of singularities of $\mathcal{C}(M)$.

Let $n \in U$ and $w \in W^{I}$. As $\mathbb{k}[\mathcal{C}(M)]^{U^{\prime}}$ is generated by $\left\{f_{i}, \tilde{f}_{i} \mid i \in M\right\}$, we have

$$
\phi^{-1}\left(n \dot{w} P_{I}^{-}\right) \cap \tau^{-1}\left(\mathfrak{N}_{U^{\prime}}(M)\right)=\left\{n \dot{w} * v \mid f_{i}(n \dot{w} \cdot v)=0, \tilde{f}_{i}(n \dot{w} \cdot v)=0 \quad \forall i \in M\right\} .
$$


Here $f_{i}$ (resp. $\tilde{f}_{i}$ ) is regarded as the coordinate of $v_{-\varpi_{i}} \in \mathrm{R}\left(\varpi_{i}^{*}\right)$ (resp. $v_{-\varpi_{i}+\alpha_{i}} \in \mathrm{R}\left(\varpi_{i}^{*}\right)$ ). Note that $f_{i}(n \dot{w} \cdot v)$ depends only on the component $v_{i}$ of $v$, and $v_{i}$ is proportional to $v_{-\varpi_{i}}$. Let us simplify condition (3.2). Since $f_{i}$ is actually a $U$-invariant, we have $f_{i}\left(n \dot{w} \cdot v_{i}\right)=$ $f_{i}\left(\dot{w} \cdot v_{i}\right)$. Next, $\tilde{f}_{i}$ is invariant with respect to a subgroup of codimension 1 in $U$. Namely, consider the decomposition $U=U^{\alpha_{i}} U_{\alpha_{i}} \simeq U^{\alpha_{i}} \times U_{\alpha_{i}}$, where $U_{\alpha_{i}}$ is the root subgroup and $U^{\alpha_{i}}$ is the unipotent radical of the minimal parabolic subgroup associated with $\alpha_{i}$. If $n_{i} \in U_{\alpha_{i}}$ and $\tilde{n} \in U^{\alpha_{i}}$, then $\tilde{n} \cdot \tilde{f}_{i}=\tilde{f}_{i}$ and $n_{i}^{-1} \cdot \tilde{f}_{i}=\tilde{f}_{i}+c_{i} f_{i}$ for some $c_{i}=c_{i}\left(n_{i}\right) \in \mathbb{k}$. Hence for $n=\tilde{n} n_{i} \in U$, we have

$$
\tilde{f}_{i}\left(n \dot{w} \cdot v_{i}\right)=\tilde{f}_{i}\left(n_{i} \dot{w} \cdot v_{i}\right)=\left(n_{i}^{-1} \cdot \tilde{f}_{i}\right)\left(\dot{w} \cdot v_{i}\right)=\tilde{f}_{i}\left(\dot{w} \cdot v_{i}\right)+f_{i}\left(\dot{w} \cdot v_{i}\right) c_{i} .
$$

Therefore, (3.2) reduces to the following:

$$
\phi^{-1}\left(n \dot{w} P_{I}^{-}\right) \cap \tau^{-1}\left(\mathfrak{N}_{U^{\prime}}(M)\right)=\left\{n \dot{w} * v \mid f_{i}\left(\dot{w} \cdot v_{i}\right)=0, \tilde{f}_{i}\left(\dot{w} \cdot v_{i}\right)=0 \quad \forall i \in M\right\} .
$$

Thus, the dimension of this intersection does not depend on $n \in U$; it depends only on $w \in W^{I}$, i.e., on $\mathcal{O}(w) \subset G / P_{I}^{-}$. We can make (3.3) more precise by using the partition of $\mathcal{C}(M)$ into (finitely many) $G$-orbits. For any subset $J \subset M$, let $\boldsymbol{v}_{J}=\sum_{i \in J} v_{-\varpi_{i}} \in V$. Then $\left\{\boldsymbol{v}_{J} \mid J \subset M\right\}$ is a complete set of representatives of the $G$-orbits in $\mathcal{C}(M)$ (Theorem 1.1(2)). Set $\stackrel{\circ}{V}_{J}=G \cdot \boldsymbol{v}_{J} \cap V=T \cdot \boldsymbol{v}_{J}$. It is an open subset of a $(\# J)$-dimensional vector space. Then $\phi^{-1}\left(n \dot{w} P_{I}^{-}\right) \cap \tau^{-1}\left(\mathfrak{N}_{U^{\prime}}(M) \cap G \cdot \boldsymbol{v}_{J}\right)=\left\{n \dot{w} * v \mid v \in \stackrel{\circ}{V}_{J}, f_{i}\left(\dot{w} \cdot v_{i}\right)=0, \tilde{f}_{i}\left(\dot{w} \cdot v_{i}\right)=0 \forall i \in M\right\}$.

This set is non-empty if and only if $\dot{w} \cdot v_{-\varpi_{i}}$ has the trivial projection to $\left\langle v_{-\varpi_{i}}, v_{-\varpi_{i}+\alpha_{i}}\right\rangle \subset$ $\mathrm{R}\left(\varpi_{i}^{*}\right)$ for all $i \in J$, i.e., $w\left(\varpi_{i}\right) \prec \varpi_{i}-\alpha_{i}$ for all $i \in J$. In this case the dimension of this set equals $\operatorname{dim} \stackrel{\circ}{V}_{J}=\# J$. Consequently, if $\phi^{-1}(\mathcal{O}(w)) \cap \tau^{-1}\left(\mathfrak{N}_{U^{\prime}}(M) \cap G \cdot \boldsymbol{v}_{J}\right) \neq \varnothing$, then

$$
\begin{gathered}
w\left(\varpi_{i}\right) \prec \varpi_{i}-\alpha_{i} \text { for all } i \in J \text { and } \\
\operatorname{dim}\left(\phi^{-1}(\mathcal{O}(w)) \cap \tau^{-1}\left(\mathfrak{N}_{U^{\prime}}(M) \cap G \cdot \boldsymbol{v}_{J}\right)\right)=\# J+\operatorname{dim} \mathcal{O}(w) .
\end{gathered}
$$

By Lemma 3.6, $\ell(w) \geqslant 2 \cdot \# J$. Therefore,

$$
\begin{aligned}
\operatorname{dim}\left(\phi^{-1}(\mathcal{O}(w))\right. & \left.\cap \tau^{-1}\left(\mathfrak{N}_{U^{\prime}}(M) \cap G \cdot \boldsymbol{v}_{J}\right)\right)= \\
& \# J-\operatorname{codim} \mathcal{O}(w)+\operatorname{dim} G / P_{I}^{-}=\# J-\ell(w)+\operatorname{dim} G / P_{I}^{-} \leqslant \operatorname{dim} G / P_{I}^{-}-\# J .
\end{aligned}
$$

This is an upper bound for the dimension of the pullback in $G \times_{P_{I}^{-}} V$ of a subset of $\mathfrak{N}_{U^{\prime}}(M)$. If $\boldsymbol{v}_{J}$ is not generic, i.e., $J \neq M$, then $\operatorname{dim} \tau^{-1}\left(\boldsymbol{v}_{J}\right)>0$ and the actual subset of $\mathfrak{N}_{U^{\prime}}(M)$ has smaller dimension. More precisely, set $\tilde{I}=\left\{\alpha_{i} \mid i \notin J\right\}$. Then $\tilde{I} \supset I$ and $\tau^{-1}\left(\boldsymbol{v}_{J}\right) \simeq P_{\tilde{I}}^{-} / P_{I}^{-}$. Since $\operatorname{srk}\left(L_{\tilde{I}}\right)=\operatorname{srk}\left(L_{I}\right)+(m-\# J)$, we have $\operatorname{dim} \tau^{-1}\left(\boldsymbol{v}_{J}\right) \geqslant m-\# J$. Thus, for all $w \in W^{I}$ and $J \subset M$, we have

$$
\begin{aligned}
\operatorname{dim}\left(\tau\left(\phi^{-1}(\mathcal{O}(w))\right) \cap \mathfrak{N}_{U^{\prime}}(M) \cap G \cdot \boldsymbol{v}_{J}\right) & \leqslant \\
& \operatorname{dim} G / P_{I}^{-}-\# J-(m-\# J)=\operatorname{dim} G / P_{I}^{-}-m,
\end{aligned}
$$


and therefore $\operatorname{dim} \mathfrak{N}_{U^{\prime}}(M) \leqslant \operatorname{dim} G / P_{I}^{-}-m$.

Remark 3.8. A "dual" approach is to consider the $P_{I}$-stable subspace $\tilde{V}=\bigoplus_{i \in M} \mathbb{k} v_{\varpi_{i}^{*}} \subset$ $\bigoplus_{i \in M} \mathrm{R}\left(\varpi_{i}^{*}\right)$ and the map $G \times_{P_{I}} \tilde{V} \rightarrow \mathcal{C}(M)$. Then one has to work with $U_{-}$-orbits in $G / P_{I}$ and $U_{-}$-invariants in $\mathbb{k}[\mathcal{C}(M)]$, but all dimension estimates remain the same. Such an approach is realised in [13, Sect. 5], where the equidimensionality problem is considered for the actions of $U$ on $\mathcal{S}$-varieties.

Combining Theorems 3.2, 3.4, and 3.7, we obtain the general criterion:

Theorem 3.9. For a finitely generated monoid $\mathfrak{S} \subset \mathfrak{X}_{+}$, the following conditions are equivalent:

(i) $\mathcal{C}(\mathfrak{S}) / / U^{\prime}$ is an affine space and $\pi_{\mathcal{C}(\mathfrak{S}), U^{\prime}}: \mathcal{C}(\mathfrak{S}) \rightarrow \mathcal{C}(\mathfrak{S}) / / U^{\prime}$ is equidimensional;

(ii) $\mathfrak{S}$ is generated by a sparse set of fundamental weights.

\section{EQUIDIMENSIONAL QUOTIENTS BY $U^{\prime}$}

In this section, the quotient morphism for the $\mathcal{S}$-variety $\mathcal{C}(\mathfrak{S})$ will be denoted by $\pi_{\mathfrak{S}, U^{\prime}}$. Similarly, for the HV-variety $\mathcal{C}(\lambda)$, we use notation $\pi_{\lambda, U^{\prime}}$. Our goal is to characterise the monoids $\mathfrak{S}$ such that $\pi_{\mathfrak{S}, U^{\prime}}: \mathcal{C}(\mathfrak{S}) \rightarrow \mathcal{C}(\mathfrak{S}) / / U^{\prime}$ is equidimensional (i.e., without assuming that $\mathcal{C}(\mathfrak{S}) / / U^{\prime}$ is an affine space). We assume that $U^{\prime} \neq\{1\}$, i.e., $G$ is not a product of several $S L_{2}$.

First, we consider the case of HV-varieties.

Theorem 4.1. For any $\lambda \in \mathfrak{X}_{+}$, the null-cone $\mathfrak{N}_{U^{\prime}}(\mathcal{C}(\lambda))$ is of codimension 2 in $\mathcal{C}(\lambda)$.

Proof. As in the proof of Theorem 3.7, we work with the diagram

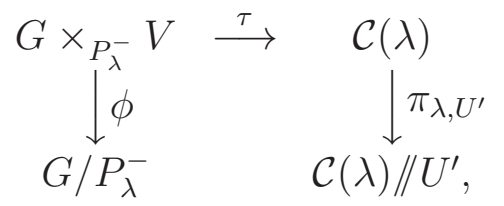

where $V=\mathbb{k} v_{-\lambda}, \phi(g * v):=g P_{\lambda}^{-}$and $\tau(g * v):=g \cdot v$. Note that $P_{\lambda}^{-}$is just the stabiliser of the line $V \subset \mathrm{R}\left(\lambda^{*}\right)$. For simplicity, we write $\mathfrak{N}_{U^{\prime}}(\lambda)$ in place of $\mathfrak{N}_{U^{\prime}}(\mathcal{C}(\lambda))$.

Since $\mathfrak{N}_{U^{\prime}}(\lambda)$ is $U$-stable, $\phi\left(\tau^{-1}\left(\mathfrak{N}_{U^{\prime}}(\lambda)\right)\right)$ is a union of $U$-orbits. Recall that $\mathbb{k}[\mathcal{C}(\lambda)]^{U^{\prime}}$ is generated by the space $\mathrm{R}(\lambda)^{U^{\prime}}$, and the corresponding set of $T$-weights is $\mathcal{J}_{\lambda}$.

We point out a $w \in W^{\lambda}$ such that the $U$-orbit $\mathcal{O}(w) \subset G / P_{\lambda}^{-}$is of codimension 2 and $\phi^{-1}(\mathcal{O}(w)) \subset \tau^{-1}\left(\mathfrak{N}_{U^{\prime}}(\lambda)\right)$. Suppose that $\left(\lambda, \alpha_{1}^{\vee}\right)=a_{1} \geqslant 1$ and $\alpha_{1}$ is a simple root of a simple component of $G$ of rank $\geqslant 2$. Let $\alpha_{2}$ be a simple root adjacent to $\alpha_{1}$ in the Dynkin diagram. Take $w=s_{2} s_{1}$. Regardless of the value of $\left(\lambda, \alpha_{2}\right)$, it is true that $w \in W^{\lambda}$ and $\ell(w)=2$. We have

$$
s_{2} s_{1}(\lambda)=\lambda-a_{1} \alpha_{1}-\left(a_{2}-a_{1}\left(\alpha_{1}, \alpha_{2}^{\vee}\right)\right) \alpha_{2} \preccurlyeq \lambda-a_{1} \alpha_{1}-\left(a_{1}+a_{2}\right) \alpha_{2},
$$


where $a_{2}=\left(\lambda, \alpha_{2}^{\vee}\right)$. Hence $s_{2} s_{1}(\lambda) \notin \mathcal{J} \lambda$. It follows that $\dot{s}_{2} \dot{s}_{1}\left(v_{-\lambda}\right) \in \mathfrak{N}_{U^{\prime}}(\lambda)$ and

$$
\tau\left(\phi^{-1}(\mathcal{O}(w))\right)=U \cdot\left(\dot{s}_{2} \dot{s}_{1}(V)\right) \in \mathfrak{N}_{U^{\prime}}(\lambda) .
$$

Thus, $w=s_{2} s_{1}$ is the required element. Since $\tau$ is injective outside the zero section of $\phi$, it is still true that $\operatorname{codim}_{\mathcal{C}(\lambda)} \tau\left(\phi^{-1}(\mathcal{O}(w))\right)=2$. This proves that codim $\mathfrak{N}_{U^{\prime}}(\lambda) \leqslant 2$.

On the other hand, the similar argument shows that if $w \in W^{\lambda}$ and $\ell(w)=1$ (i.e., $w=s_{i}$, where $\left.\left(\alpha_{i}, \lambda\right) \neq 0\right)$, then $\dot{w} \cdot v_{-\lambda} \notin \mathfrak{N}_{U^{\prime}}(\lambda)$. Therefore, codim $\mathfrak{N}_{U^{\prime}}(\lambda)=2$.

Corollary 4.2. Suppose that $U^{\prime} \neq\{1\}$. Then $\pi_{\lambda, U^{\prime}}: \mathcal{C}(\lambda) \rightarrow \mathcal{C}(\lambda) / / U^{\prime}$ is equidimensional if and only if $\lambda=a_{i} \varpi_{i}$ for some $i$. In particular, if the action of $G$ on $\mathcal{C}(\lambda)$ is effective and $\pi_{\lambda, U^{\prime}}$ is equidimensional, then $G$ is simple.

Proof. It follows from Theorem 2.2(i) that $\operatorname{dim} \mathcal{C}(\lambda) / / U^{\prime}=2$ if and only if $\lambda=a_{i} \varpi_{i}$.

Now, we turn to considering general monoids $\mathfrak{S} \subset \mathfrak{X}_{+}$. For any $S \subset \mathfrak{X}$, let $\operatorname{con}(S)$ denote the closed cone in $\mathfrak{X}_{\mathbb{R}}$ generated by $S$.

Lemma 4.3. Suppose that we are given two monoids $\mathfrak{S}_{1}$ and $\mathfrak{S}_{2}$ such that $\operatorname{con}\left(\mathfrak{S}_{1}\right)=\operatorname{con}\left(\mathfrak{S}_{2}\right)$. Then $\pi_{\mathfrak{S}_{1}, U^{\prime}}$ is equidimensional if and only if $\pi_{\mathfrak{S}_{2}, U^{\prime}}$ is.

Proof. It suffices to treat the case in which $\mathfrak{S}_{2}=\operatorname{con}\left(\mathfrak{S}_{1}\right) \cap \mathfrak{X}_{+}$. Then $\mathbb{k}\left[\mathcal{C}\left(\mathfrak{S}_{2}\right)\right]$ is a finite $\mathbb{k}\left[\mathcal{C}\left(\mathfrak{S}_{1}\right)\right]$-module [22, Prop. 4]. Consider the commutative diagram

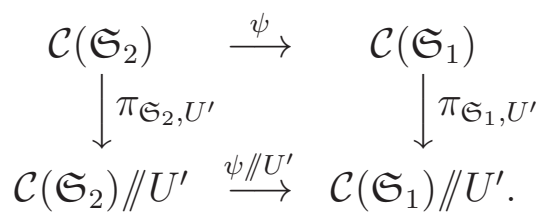

Here $\psi$ is finite, and it suffices to prove that $\psi / / U^{\prime}$ is also finite, i.e., that $\mathbb{k}\left[\mathcal{C}\left(\mathfrak{S}_{2}\right)\right]^{U^{\prime}}$ is a finite $\mathbb{k}\left[\mathcal{C}\left(\mathfrak{S}_{1}\right)\right]^{U^{\prime}}$-module. By the "transfer principle" $([2, \mathrm{Ch} .1],[15, \S 3])$, we have

$$
\mathbb{k}[X]^{U^{\prime}} \simeq\left(\mathbb{k}[X] \otimes \mathbb{k}\left[G / U^{\prime}\right]\right)^{G}
$$

for any affine $G$-variety $X$. Hence, one has to prove that $\left(\mathbb{k}\left[\mathfrak{S}_{2}\right] \otimes \mathbb{k}\left[G / U^{\prime}\right]\right)^{G}$ is a finite $\left(\mathbb{k}\left[\mathfrak{S}_{1}\right] \otimes \mathbb{k}\left[G / U^{\prime}\right]\right)^{G}$-module, which readily follows from the fact that $\mathbb{k}\left[G / U^{\prime}\right]$ is finitely generated and $G$ is reductive.

Theorem 4.4. The quotient morphism $\pi_{\mathfrak{S}, U^{\prime}}$ is equidimensional if and only if $\operatorname{con}(\mathfrak{S})$ is generated by a sparse set of fundamental weights.

Proof. 1) The "if" part readily follows from Lemma 4.3 and Theorem 3.7.

2) Suppose that $\pi_{\mathfrak{S}, U^{\prime}}: \mathcal{C}(\mathfrak{S}) \rightarrow \mathcal{C}(\mathfrak{S}) / / U^{\prime}$ is equidimensional. By Lemma 4.3 , it suffices to consider the case in which $\mathfrak{S}=\operatorname{con}(\mathfrak{S}) \cap \mathfrak{X}_{+}$. Then $\mathcal{C}(\mathfrak{S})$ is normal (see Theorem 1.1(3)). Consider an arbitrary edge, $\operatorname{con}(\lambda)$, of $\operatorname{con}(\mathfrak{S})$. It is assumed that $\lambda \in \mathfrak{S}$ is a primitive element of $\mathfrak{X}_{+}$. By [22, Prop.7], the HV-variety $\mathcal{C}(\lambda)$ is a subvariety of $\mathcal{C}(\mathfrak{S})$. On the other 
hand, $\mathbb{k}[\mathcal{C}(\lambda)]=\bigoplus_{n \geqslant 0} \mathrm{R}(n \lambda)$ is a $G$-stable subalgebra of $\mathbb{k}[\mathcal{C}(\mathfrak{S})]=\bigoplus_{\mu \in \mathfrak{S}} \mathrm{R}(\mu)$. This yields the chain of $G$-equivariant maps

$$
\mathcal{C}(\lambda) \hookrightarrow \mathcal{C}(\mathfrak{S}) \stackrel{r}{\longrightarrow} \mathcal{C}(\lambda) .
$$

Here the composite map is the identity, i.e., $r$ is a $G$-equivariant retraction. Furthermore, passage to the subalgebras of $U^{\prime}$-invariants (= quotient varieties) yields the maps

$$
\mathcal{C}(\lambda) / / U^{\prime} \hookrightarrow \mathcal{C}(\mathfrak{S}) / / U^{\prime} \stackrel{r / U^{\prime}}{\longrightarrow} \mathcal{C}(\lambda) / / U^{\prime},
$$

which shows that $r / / U^{\prime}$ is a retraction, too. This also shows that both $r$ and $r / / U^{\prime}$ are onto. Consider the commutative diagram

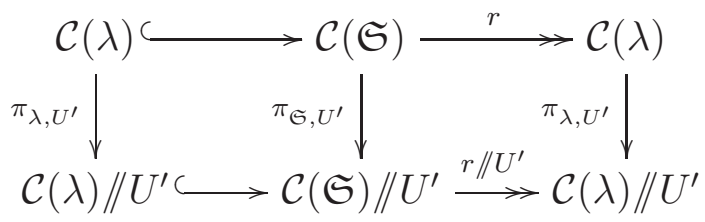

As $\mathcal{C}(\mathfrak{S})$ is normal, the same is true for $\mathcal{C}(\mathfrak{S}) / / U^{\prime}$. Since $\pi_{\mathfrak{S}, U^{\prime}}$ is equidimensional and both $\mathcal{C}(\mathfrak{S})$ and $\mathcal{C}(\mathfrak{S}) / / U^{\prime}$ are conical, it follows from Lemma 1.5 that $\pi_{\mathfrak{S}, U^{\prime}}$ is onto. Therefore, $\pi_{\lambda, U^{\prime}}$ is onto as well. Furthermore, $\pi_{\lambda, U^{\prime}}=\left.\pi_{\mathfrak{S}, U^{\prime}}\right|_{\mathcal{C}(\lambda)}$, since $\mathcal{C}(\lambda)$ is a $G$-stable subvariety of $\mathcal{C}(\mathfrak{S})$. This shows that $\pi_{\mathfrak{S}, U^{\prime}}(\mathcal{C}(\lambda))$ is a closed subset of $\mathcal{C}(\mathfrak{S}) / / U^{\prime}$.

Let $Y \subset \mathcal{C}(\mathfrak{S})$ be an irreducible component of $\pi_{\mathfrak{S}, U^{\prime}}^{-1}\left(\pi_{\mathfrak{S}, U^{\prime}}(\mathcal{C}(\lambda))\right)$ that contains $\mathcal{C}(\lambda)$ and maps dominantly to $\pi_{\mathfrak{S}, U^{\prime}}(\mathcal{C}(\lambda))$. Consider the commutative diagram

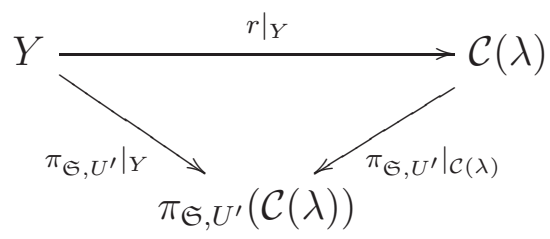

By the very construction of $Y$, the morphism $\left.r\right|_{Y}$ is onto and $\left.\pi_{\mathfrak{S}, U^{\prime}}\right|_{Y}$ is equidimensional. It follows that $\left.\pi_{\mathfrak{S}, U^{\prime}}\right|_{\mathcal{C}(\lambda)}$ is also equidimensional. Consequently, $\pi_{\lambda, U^{\prime}}=\left.\pi_{\mathfrak{S}, U^{\prime}}\right|_{\mathcal{C}(\lambda)}$ is equidimensional and, by Corollary $4.2, \lambda=\varpi_{i}$ for some $i$ (recall that $\lambda$ is supposed to be primitive). Thus, the edges of con(S) are generated by fundamental weights. Finally, by Theorem 3.4, the corresponding set of fundamental weights is sparse.

Remark 4.5. Our proof of the "only if" part exploits ideas of Vinberg and Wehlau for the equidimensional quotients by $G$ (see [23, Theorem 8.2] and [24, Prop. 2.6]).

Remark 4.6. We can prove a general equidimensionality criterion for the quotients of $\mathcal{S}$ varieties by $U$. This topic will be considered in a forthcoming publication. 


\section{THE HILBERT-MUMFORD CRITERION FOR $U^{\prime}$}

Let $X$ be an irreducible affine $G$-variety and $x_{0} \in X^{G}$. For any $H \subset G$, define the null-cone with respect to $H$ and $x_{0}$ as

$$
\mathfrak{N}_{H}(X)=\left\{x \in X \mid F(x)=F\left(x_{0}\right) \quad \forall F \in \mathbb{k}[X]^{H}\right\} .
$$

If $\mathbb{k}[X]^{H}$ is finitely generated, then $\mathfrak{N}_{H}(X)$ can be regarded as the fibre of $\pi_{X, H}$ containing $x_{0}$. Below, we give a characterisation of $\mathfrak{N}_{U^{\prime}}(X)$ via one-parameter subgroups (1-PS for short) of $T$. This is inspired by Brion's description of null-cones for $U$-invariants [3, Sect. IV]. Recall that the Hilbert-Mumford criterion for $G$ asserts that

$x \in \mathfrak{N}_{G}(X)$ if and only if there is a 1-PS $\tau: \mathbb{k}^{\times} \rightarrow G$ such that $\lim _{t \rightarrow 0} \tau(t) \cdot x=x_{0}$ (cf. [9, III.2], [23, §5.3]). By [14, Theorem 2.2], there is the canonical affine model of the homogeneous space $G / U^{\prime}$, that is, an affine pointed $G$-variety $\left(\overline{G / U^{\prime}}, \boldsymbol{p}\right)$ such that

- $G_{p}=U^{\prime}$;

- $G \cdot \boldsymbol{p}$ is dense in $\overline{G / U^{\prime}}$;

- $\mathbb{k}\left[\overline{G / U^{\prime}}\right]=\mathbb{k}[G]^{U^{\prime}}$.

Here $\boldsymbol{p}=\left(f_{1}, \tilde{f}_{1}, \ldots, f_{r}, \tilde{f}_{r}\right)$ is a direct sum of weight vectors in $2 \mathrm{R}\left(\varpi_{1}\right) \oplus \cdots \oplus 2 \mathrm{R}\left(\varpi_{r}\right)$, with weights $\varpi_{i}, \varpi_{i}-\alpha_{i}(1 \leqslant i \leqslant r)$. If $G$ has no simple factors $S L_{2}, S L_{3}$, then all these weights belong to an open half-space of $\mathfrak{X}_{\mathbb{R}}$ (see the proof of [14, Prop. 1.9]). In this case, $\boldsymbol{p}$ is unstable and $\overline{G / U^{\prime}}$ contains the origin in $2 \mathrm{R}\left(\varpi_{1}\right) \oplus \cdots \oplus 2 \mathrm{R}\left(\varpi_{r}\right)$. Let $\tau: \mathbb{k}^{\times} \rightarrow T$ be a 1-PS. Using the canonical pairing between $\mathfrak{X}$ and the set of 1-PS of $T$, we will regard $\tau$ as an element of $\mathfrak{X}_{\mathbb{R}}$. Let us say that $\tau$ is $U^{\prime}$-admissible, if $\left(\tau, \varpi_{i}\right)>0$ and $\left(\tau, \varpi_{i}-\alpha_{i}\right)>0$ for all $i$; that is, if $\lim _{t \rightarrow 0} \tau(t) \cdot \boldsymbol{p}=0$. Since $\mathbb{k}\left[\overline{G / U^{\prime}}\right]=\mathbb{k}[G]^{U^{\prime}}$, one has the isomorphism

$$
\mathbb{k}\left[X \times \overline{G / U^{\prime}}\right]^{G}=\left(\mathbb{k}[X] \otimes \mathbb{k}[G]^{U^{\prime}}\right)^{G} \stackrel{\sim}{\longrightarrow} \mathbb{k}[X]^{U^{\prime}}
$$

that takes $\tilde{F}(\cdot, \cdot) \in \mathbb{k}\left[X \times \overline{G / U^{\prime}}\right]^{G}$ to $F(\cdot)=\tilde{F}(\cdot, \boldsymbol{p}) \in \mathbb{k}[X]^{U^{\prime}}$.

Theorem 5.1. Suppose that $G$ has no simple factors $S L_{2}, S L_{3}$. Then the following conditions are equivalent:

(i) $x \in \mathfrak{N}_{U^{\prime}}(X)$, i.e., $F(x)=F\left(x_{0}\right)$ for all $F \in \mathbb{k}[X]^{U^{\prime}}$;

(ii) there is $u \in U$ and a $U^{\prime}$-admissible 1-PS $\tau: \mathbb{k}^{\times} \rightarrow T$ such that $\lim _{t \rightarrow 0} \tau(t) u \cdot x=x_{0}$.

Proof. (i) $\Rightarrow$ (ii). Suppose that $x \in \mathfrak{N}_{U^{\prime}}(X)$. Then $\tilde{F}(x, \boldsymbol{p})=F(x)=F\left(x_{0}\right)=\tilde{F}\left(x_{0}, \boldsymbol{p}\right)$. Since $\boldsymbol{p}$ is unstable in $\overline{G / U^{\prime}}$, we have $\tilde{F}\left(x_{0}, \boldsymbol{p}\right)=\tilde{F}\left(x_{0}, 0\right)$. Thus, $\tilde{F}(x, \boldsymbol{p})=\tilde{F}\left(x_{0}, 0\right)$ for all $\tilde{F} \in\left(\mathbb{k}[X] \otimes \mathbb{k}[G]^{U^{\prime}}\right)^{G}$, i.e., $(x, \boldsymbol{p}) \in \mathfrak{N}_{G}\left(X \times \overline{G / U^{\prime}}\right)$. By the Hilbert-Mumford criterion for $G$, there is a $1-\mathrm{PS} \nu: \mathbb{k}^{\times} \rightarrow G$ such that $\nu(t) \cdot(x, \boldsymbol{p}) \underset{t \rightarrow 0}{\longrightarrow}\left(x_{0}, 0\right)$.

By a result of Grosshans [10, Cor. 1] (see also [3, IV.1]), we may assume that $\nu\left(\mathbb{k}^{\times}\right) \subset B$. Then there is $u \in U$ such that $\tau(t):=u \nu(t) u^{-1} \in T$. Therefore,

$$
\tau(t) u \cdot(x, \boldsymbol{p}) \underset{t \rightarrow 0}{\longrightarrow}\left(x_{0}, 0\right) .
$$


Note that $u \cdot \boldsymbol{p}(u \in U)$ does not differ much from $\boldsymbol{p}$. Namely, each component $f_{i}$ remains intact, whereas $\tilde{f}_{i}$ is replaced with $\tilde{f}_{i}+c_{i} f_{i}$ for some $c_{i} \in \mathbb{k}$. This means that $\tau(t) u \cdot \boldsymbol{p} \underset{t \rightarrow 0}{\longrightarrow} 0$ if and only if $\tau(t) \cdot \boldsymbol{p} \underset{t \rightarrow 0}{\longrightarrow} 0$. That is, $\tau$ is actually $U^{\prime}$-admissible and $\lim _{t \rightarrow 0} \tau(t) u \cdot x=x_{0}$.

(ii) $\Rightarrow$ (i). Suppose that $F \in \mathbb{k}[X]^{U^{\prime}}$ and $\tilde{F}$ is the corresponding $G$-invariant in $\mathbb{k}\left[X \times \overline{G / U^{\prime}}\right]$. Then $F(x)=\tilde{F}(x, \boldsymbol{p})=\tilde{F}(\tau(t) u \cdot x, \tau(t) u \cdot \boldsymbol{p})$. Since $u \cdot \boldsymbol{p}$ is a linear combination of weight vectors with the same weights and $\tau$ is $U^{\prime}$-admissible, we have $\lim _{t \rightarrow 0} \tau(t) u \cdot \boldsymbol{p}=0$. Hence $F(x)=\tilde{F}\left(x_{0}, 0\right)=\tilde{F}\left(x_{0}, \boldsymbol{p}\right)=F\left(x_{0}\right)$.

Remark 5.2. Our Theorem 5.1 is similar to Theorem 5 in [3] on null-cones for $U$-invariants. The only difference is that we end up with a smaller class of admissible 1-PS.

Obviously, there are inclusions $\mathfrak{N}_{U^{\prime}}(X) \subset \mathfrak{N}_{U}(X) \subset \mathfrak{N}_{G}(X)$ and hence

$$
G \cdot \mathfrak{N}_{U^{\prime}}(X) \subset G \cdot \mathfrak{N}_{U}(X) \subset \mathfrak{N}_{G}(X) .
$$

It is proved in [3, Théorème 6(ii)] that actually $G \cdot \mathfrak{N}_{U}(X)=\mathfrak{N}_{G}(X)$. Below, we investigate the similar problem for $U^{\prime}$.

Recall that $\operatorname{con}(S)$ is the closed cone in $\mathfrak{X}_{\mathbb{R}}$ generated by $S$. If $K \subset \mathfrak{X}_{\mathbb{R}}$ is a closed cone, then $K^{\perp}$ denotes the dual cone and $K^{o}$ denotes the relative interior of $K$. By the very definition, the cone generated by the $U^{\prime}$-admissible 1-PS is open, and its closure is dual to $\operatorname{con}\left(\left\{\varpi_{i}, \varpi_{i}-\alpha_{i} \mid i=1, \ldots, r\right\}\right)$. By [14, Theorem 4.2], we have

$$
\operatorname{con}\left(\left\{\varpi_{i}, \varpi_{i}-\alpha_{i} \mid i=1, \ldots, r\right\}\right)^{\perp}=\operatorname{con}\left(\Delta^{+} \backslash \Pi\right) .
$$

Hence the cone generated by the $U^{\prime}$-admissible 1-PS equals $\operatorname{con}\left(\Delta^{+} \backslash \Pi\right)^{o}$.

Theorem 5.3. Suppose that $G$ has no simple factors of type $S L$. Then

1) $\operatorname{con}\left(\varpi_{1}, \ldots, \varpi_{r}\right) \subset \operatorname{con}\left(\Delta^{+} \backslash \Pi\right)$,

2) $G \cdot \mathfrak{N}_{U^{\prime}}(X)=\mathfrak{N}_{G}(X)$ for all affine $G$-varieties $X$.

Proof. 1) Taking the dual cones yields the equivalent condition that

$$
\operatorname{con}\left(\left\{\varpi_{i}, \varpi_{i}-\alpha_{i} \mid i=1, \ldots, r\right\}\right) \subset \operatorname{con}\left(\Delta^{+}\right) .
$$

That is, one has to verify that each $\varpi_{i}-\alpha_{i}$ has non-negative coefficients in the expression via the simple roots. Let $C$ denote the Cartan matrix of a simple group $G$. All the entries of $C^{-1}$ are positive and the rows of $C^{-1}$ provide the expressions of the fundamental weights via the simple roots. Hence it remains to check that the diagonal entries of $C^{-1}$ are $\geqslant 1$. An explicit verification shows that this is true if $G \neq S L_{r+1}$. (The matrices $C^{-1}$ can be found in [21, Table 2].)

2) Suppose that $x \in \mathfrak{N}_{G}(X)$. Then there exist $g \in G$ and $\tau: \mathbb{k}^{\times} \rightarrow T$ such that $\lim _{t \rightarrow 0} \tau(t) g \cdot x=x_{0}$. Let $y=g \cdot x$. The set of all 1-PS $\nu: \mathbb{k}^{\times} \rightarrow T$ such that $\lim _{t \rightarrow 0} \nu(t) \cdot y=x_{0}$ generates an open cone in $\mathfrak{X}_{\mathbb{R}}$. Therefore, we may assume that $\tau$ is a regular 1-PS. 
Now, in view of the Hilbert-Mumford criterion for $G$ and Theorem 5.1, it suffices to prove that any regular 1-PS of $T$ is $W$-conjugate to a $U^{\prime}$-admissible one. This follows from part 1$)$, since $\operatorname{con}\left(\varpi_{1}, \ldots, \varpi_{r}\right)$ is a fundamental domain for the $W$-action on $\mathfrak{X}_{\mathbb{R}}$ and $\operatorname{con}\left(\varpi_{1}, \ldots, \varpi_{r}\right)^{o} \subset \operatorname{con}\left(\Delta^{+} \backslash \Pi\right)^{o}$.

For $G=S L_{r+1}$, we have $\varpi_{1}-\alpha_{1}, \varpi_{r}-\alpha_{r} \notin \operatorname{con}\left(\Delta^{+}\right)$and therefore, $\operatorname{con}\left(\varpi_{1}, \ldots, \varpi_{r}\right) \not \subset$ $\operatorname{con}\left(\Delta^{+} \backslash \Pi\right)$. More precisely, $\varpi_{1}, \varpi_{r} \not \subset \operatorname{con}\left(\Delta^{+} \backslash \Pi\right)$. This means that one may expect that, for some $S L_{r+1}$-varieties, there is the strict inclusion $G \cdot \mathfrak{N}_{U^{\prime}}(X) \varsubsetneqq \mathfrak{N}_{G}(X)$.

Example 5.4. For $m \geqslant 3$, consider the representation of $G=S L_{3}$ in the space $V=\mathrm{R}\left(m \varpi_{1}\right)$ of forms of degree $m$ in three variables $x, y, z$. By Theorem 1.2, $\operatorname{dim} V^{U^{\prime}}=m+1$. Let $U$ be the subgroup of the unipotent upper-triangular matrices in the basis dual to $(x, y, z)$. The $U^{\prime}$-invariants of degree 1 are the coefficients of $x^{m}, x^{m-1} y, \ldots, x y^{m-1}, y^{m}$. Therefore, $\mathfrak{N}_{U^{\prime}}(V)$ is contained in the subspace of forms having the linear factor $z$ and all the forms in $S L_{3} \cdot \mathfrak{N}_{U^{\prime}}(V)$ have a linear factor. On the other hand, the null-form (with respect to $S L_{3}$ ) $x^{m}+y^{m-1} z$ is irreducible. Hence, $S L_{3} \cdot \mathfrak{N}_{U^{\prime}}(V) \neq \mathfrak{N}_{S L_{3}}(V)$.

Remark. In view of Theorem 5.1, it would be much more instructive to have such an example for $S L_{n}, n \geqslant 4$. However, we are unable to provide it yet.

\section{EQUIDIMENSIONAL QUOTIENTS AND IRREDUCIBLE REPRESENTATIONS OF SIMPLE}

\section{GROUPS}

In this section, we transform the criterion of Theorem 3.9 in a sufficient condition applicable to a wider class of $G$-varieties. Then we obtain the list of irreducible representations $V$ of simple algebraic groups $G \neq S L_{2}$ such that $\mathbb{k}[V]$ is a free $\mathbb{k}[V]^{U^{\prime}}$-module.

For any affine irreducible $G$-variety $Z$, there is a flat degeneration $\mathbb{k}[Z] \leadsto \operatorname{gr}(\mathbb{k}[Z])$. (Brion attributes this to Domingo Luna in his thesis, see [2, Lemma 1.5]). Here $\operatorname{gr}(\mathbb{k}[Z])$ is again a finitely generated $\mathbb{k}$-algebra and a locally-finite $G$-module, and $\operatorname{gr} Z:=$ $\operatorname{Spec}(\operatorname{gr}(\mathbb{k}[Z]))$ is an affine horospherical $G$-variety. The whole theory of "contractions of actions of reductive groups" is later developed in [15]. (See also [4], [19], [11] for related results and other applications.) The "contraction" $Z \leadsto \operatorname{gr} Z$ has the property that the algebras $\mathbb{k}[Z]$ and $\mathbb{k}[\operatorname{gr} Z]=\operatorname{gr}(\mathbb{k}[Z])$ are isomorphic as $G$-modules. But the multiplication in $\mathbb{k}[\mathrm{gr} Z]$ is simpler than that in $\mathbb{k}[Z]$; namely, if $M$ and $N$ are two simple $G$-modules in $\mathbb{k}[\operatorname{gr} Z]$, then $M \cdot N$ (the product in $\mathbb{k}[\operatorname{gr} Z]$ ) is again a simple $G$-module. Furthermore, $\mathbb{k}[\operatorname{gr} Z]^{U} \simeq \mathbb{k}[Z]^{U}$ and $G \cdot\left((\operatorname{gr} Z)^{U}\right)=\operatorname{gr} Z$. This means that if $Z$ is a spherical $G$-variety, then $\operatorname{gr} Z$ is an $\mathcal{S}$-variety.

Theorem 6.1. Suppose that $G$ acts on an irreducible affine variety $X$ such that (1) $\mathbb{k}[X]^{U}$ is a polynomial algebra and (2) the weights of free generators are fundamental, different and form 
a sparse set. Then $\mathbb{k}[X]^{U^{\prime}}$ is also polynomial, of Krull dimension $2 \operatorname{dim} X / / U$, and the quotient $\pi_{X, U^{\prime}}: X \rightarrow X / / U^{\prime}$ is equidimensional.

Proof. The idea is the same as in the proof of the similar result for $U$-invariants in [13, Theorem 5.5]. We use the fact that in our situation $\operatorname{gr} X$ is an $\mathcal{S}$-variety whose monoid of dominant weights is generated by a sparse set of fundamental weights.

Let $\varpi_{1}, \ldots, \varpi_{m}$ be the weights of free generators of $\mathbb{k}[X]^{U}$. Set $\Gamma=\sum_{i=1}^{m} \mathbb{N}_{i}$. It follows from the hypotheses on weights that $\mathbb{k}[X]$ is a multiplicity free $G$-module, i.e., $X$ is a spherical $G$-variety [18, Theorem 2]. Therefore, $\mathbb{k}[X]$ is isomorphic to $\bigoplus_{\lambda \in \Gamma} \mathrm{R}(\lambda)$ as $G$ module and $\operatorname{gr} X \simeq \mathcal{C}(\Gamma)$.

By $[15, \S 5]$, there exists a $G$-variety $Y$ and a function $q \in \mathbb{k}[Y]^{G}$ such that $\mathbb{k}[Y] /(q-a) \simeq$ $\mathbb{k}[X]$ for all $a \in \mathbb{k}^{\times}, \mathbb{k}[Y]\left[q^{-1}\right] \simeq \mathbb{k}[X]\left[q, q^{-1}\right]$, and $\mathbb{k}[Y] /(q) \simeq \mathbb{k}[\operatorname{gr} X]$. Recall some details on constructing $Y$ and $\operatorname{gr} X$. Let $\varrho$ be the half-sum of the positive coroots. For $\lambda \in \mathfrak{X}_{+}$, we set ht $(\lambda)=(\lambda, \varrho)$. Letting $\mathbb{k}[X]_{(n)}=\bigoplus_{\lambda: \text { ht }(\lambda) \leqslant n} \mathrm{R}(\lambda)$, one obtains an ascending filtration of the algebra $\mathbb{k}[X]$ :

$$
\{0\} \subset \mathbb{k}[X]_{(0)} \subset \mathbb{k}[X]_{(1)} \subset \cdots \subset \mathbb{k}[X]_{(n)} \cdots .
$$

Each subspace $\mathbb{k}[X]_{(n)}$ is $G$-stable and finite-dimensional and $\mathbb{k}[X]_{(0)}=\mathbb{k}[X]^{G}=\mathbb{k}$. Let $q$ be a formal variable. Then the algebras $\mathbb{k}[Y]$ and $\operatorname{gr}(\mathbb{k}[X])$ are defined as follows:

$$
\begin{gathered}
\mathbb{k}[Y]=\bigoplus_{n=0}^{\infty} \mathbb{k}[X]_{(n)} q^{n} \subset \mathbb{k}[X][q], \\
\operatorname{gr}(\mathbb{k}[X])=\bigoplus_{n \geqslant 0} \mathbb{k}[X]_{(n)} / \mathbb{k}[X]_{(n-1)} .
\end{gathered}
$$

Let $f_{1}, \ldots, f_{m}$ be the free generators of $\mathbb{k}[X]^{U}$, where $f_{i} \in \mathrm{R}\left(\varpi_{i}\right)^{U}$, as usual. They can also be regarded as free generators of $\mathbb{k}[\operatorname{gr} X]^{U}$. By Theorem $1.4, \mathbb{k}[\operatorname{gr} X]^{U^{\prime}}$ is freely generated by $f_{1}, \tilde{f}_{1}, \ldots, f_{m}, \tilde{f}_{m}$ and by Theorem $3.9, \pi_{\mathrm{gr} X, U^{\prime}}: \operatorname{gr} X \rightarrow(\operatorname{gr} X) / / U^{\prime}$ is equidimensional. On the other hand, it follows from [14, Theorem 2.4] that $f_{1}, \tilde{f}_{1}, \ldots, f_{m}, \tilde{f}_{m}$ also generate $\mathbb{k}[X]^{U^{\prime}}$. Therefore, to conclude that $\mathbb{k}[X]^{U^{\prime}}$ is polynomial, it suffices to know that $\operatorname{dim} X / / U^{\prime}=\operatorname{dim}(\operatorname{gr} X) / / U^{\prime}(=2 m)$. To this end, we exploit the following facts:

a) For an irreducible $G$-variety $X$, there always exists a generic stabiliser for the $U$-action on $X$ [5, Corollaire 1.6], which we denote by g.s. $(U: X)$;

b) If $X$ is affine, then this generic stabiliser depends only on the $G$-module structure of $\mathbb{k}[X]$, i.e., on the highest weights of $G$-modules occurring in $\mathbb{k}[X]$ [12, Theorem 1.2.9]. Consequently, g.s. $(U: X)=$ g.s. $(U: \operatorname{gr} X)$;

c) the minimal dimension of $U^{\prime}$-stablisers in $X$ equals $\operatorname{dim}\left(U^{\prime} \cap\right.$ g.s. $\left.(U: X)\right)$ [14, Lemma 2.5]. Therefore it is the same for $X$ and $\operatorname{gr} X$;

d) Since $U^{\prime}$ is unipotent, we have $\operatorname{dim} X / / U^{\prime}=\operatorname{dim} X-\operatorname{dim} U^{\prime}+\min _{x \in X} \operatorname{dim} U_{x}^{\prime}$. 
Combining a)-d) yields the desired equality and thereby the assertion that $\mathbb{k}[X]^{U^{\prime}}$ is polynomial, of Krull dimension $2 m=2 \operatorname{dim} X / / U$.

Let $n_{i}$ be the smallest integer such that $\mathrm{R}\left(\varpi_{i}\right) \subset \mathbb{k}[X]_{\left(n_{i}\right)}$. Using the above description of $\mathbb{k}[Y]$ and $\mathbb{k}[\operatorname{gr} X]^{U^{\prime}}$, one easily obtains that

$$
\begin{gathered}
\mathbb{k}[Y]^{U}=\mathbb{k}\left[q, q^{n_{1}} f_{1}, \ldots, q^{n_{m}} f_{m}\right] \\
\mathbb{k}[Y]^{U^{\prime}}=\mathbb{k}\left[q, q^{n_{1}} f_{1}, q^{n_{1}} \tilde{f}_{1}, \ldots, q^{n_{m}} f_{m}, q^{n_{m}} \tilde{f}_{m}\right],
\end{gathered}
$$

i.e., both algebras are polynomial, of Krull dimension $m+1$ and $2 m+1$, respectively. By a result of Kraft, the first equality implies that $Y$ has rational singularities (see [2, Theorem 1.6], [15, Theorem 6]). One has the following commutative diagram:

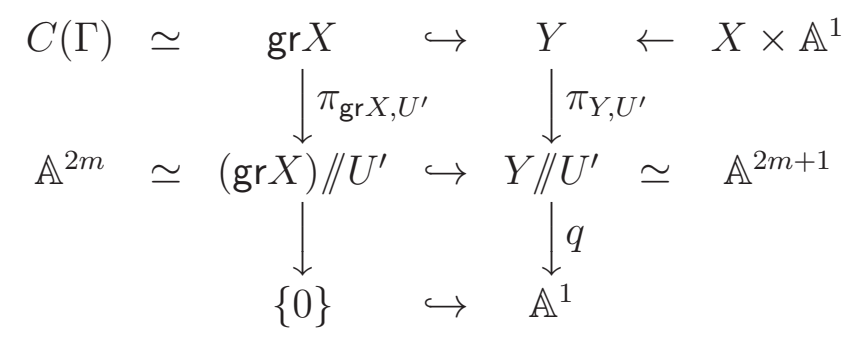

Consequently,

$$
\mathfrak{N}_{U^{\prime}}(\operatorname{gr} X)=\pi_{\operatorname{gr} X, U^{\prime}}^{-1}\left(\pi_{\operatorname{gr} X, U^{\prime}}(\overline{0})\right)=\pi_{Y, U^{\prime}}^{-1}\left(\pi_{Y, U^{\prime}}(\overline{0})\right)=\mathfrak{N}_{U^{\prime}}(Y),
$$

where $\overline{0} \in \operatorname{gr} X \subset Y$ is the unique $G$-fixed point $\operatorname{of} \operatorname{gr} X$. Since $\operatorname{dim} Y=\operatorname{dim} X+1$, $\operatorname{dim} Y / / U^{\prime}=\operatorname{dim}(\operatorname{gr} X) / / U^{\prime}+1$, and $\pi_{\mathrm{gr} X, U^{\prime}}$ is equidimensional, the morphism $\pi_{Y, U^{\prime}}$ is equidimensional as well. As $Y$ has rational singularities and hence is Cohen-Macaulay, this implies that $\mathbb{k}[Y]$ is a flat $\mathbb{k}[Y]^{U^{\prime}}$-module. Since $\mathbb{k}[Y]\left[q^{-1}\right] \simeq \mathbb{k}[X]\left[q, q^{-1}\right]$ and $\left.\mathbb{k}[Y]^{U^{\prime}}\left[q^{-1}\right] \simeq \mathbb{k}[X]\right]^{U^{\prime}}\left[q, q^{-1}\right]$, we conclude that $\mathbb{k}[X]$ is a flat $\mathbb{k}[X]^{U^{\prime}}$-module. Thus, $\pi_{X, U^{\prime}}$ is equidimensional.

Our next goal is to obtain the list of all irreducible representations $V$ of simple algebraic groups such that $\mathbb{k}[V]$ is a free $\mathbb{k}[V]^{U^{\prime}}$-module. As is well known, $\mathbb{k}[V]$ is a free $\mathbb{k}[V]^{U^{\prime}}$ module if and only if $\mathbb{k}[V]^{U^{\prime}}$ is polynomial and $\pi_{V, U^{\prime}}$ is equidimensional [16, Prop. 17.29]. Therefore, the required representations are contained in [14, Table1] and our task is to pick from that table the representations having the additional property that $\pi_{V, U^{\prime}}$ is equidimensional. The numbering of fundamental weights of simple algebraic groups follows [21, Tables].

Theorem 6.2. Let $G$ be a connected simple algebraic group with $\mathrm{rk} G \geqslant 2$ and $\mathrm{R}(\lambda)$ a simple $G$-module. The following conditions are equivalent:

(i) $\mathbb{k}[R(\lambda)]$ is a free $\mathbb{k}[R(\lambda)]^{U^{\prime}}$-module; 
(ii) Up to symmetries of the Dynkin diagram of $G$, the pairs $(G, \lambda)$ occur in the following list:

$$
\begin{aligned}
& \left(\mathbf{A}_{r}, \varpi_{1}\right),\left(\mathbf{B}_{r}, \varpi_{1}\right),\left(\mathbf{C}_{r}, \varpi_{1}\right), r \geqslant 2 ; \\
& \left(\mathbf{D}_{r}, \varpi_{1}\right), r \geqslant 3 ; \\
& \left(\mathbf{B}_{3}, \varpi_{3}\right),\left(\mathbf{B}_{4}, \varpi_{4}\right),\left(\mathbf{D}_{5}, \varpi_{5}\right),\left(\mathbf{E}_{6}, \varpi_{1}\right),\left(\mathbf{G}_{2}, \varpi_{1}\right) .
\end{aligned}
$$

Proof. (ii) $\Rightarrow($ i). By [14, Theorem 5.1], all these representations have a polynomial algebra of $U^{\prime}$-invariants. Consider $X=\mathfrak{N}_{G}(\mathrm{R}(\lambda))$, the null-cone with respect to $G$. The nonzero weights of generators of $\mathbb{k}[\mathrm{R}(\lambda)]^{U}$ (and hence the weights of generators of $\mathbb{k}[X]^{U}$ ) given by Brion [3, p. 13] are fundamental and form a sparse set. Consequently, Theorem 6.1 applies to $X$, and $\pi_{X, U^{\prime}}$ is equidimensional. Since $X$ is either a $G$-invariant hypersurface in $\mathrm{R}(\lambda)$ or equal to $\mathrm{R}(\lambda), \pi_{\mathrm{R}(\lambda), U^{\prime}}$ is also equidimensional.

(i) $\Rightarrow$ (ii). We have to prove that, for the other items in [14, Table1], the quotient is not equidimensional. The list of such "bad" pairs $(G, \lambda)$ is: $\left(\mathbf{A}_{r}, \varpi_{2}^{*}\right)$ with $r \geqslant 4 ;\left(\mathbf{B}_{5}, \varpi_{5}\right)$, $\left(\mathbf{D}_{6}, \varpi_{6}\right),\left(\mathbf{E}_{7}, \varpi_{1}\right),\left(\mathbf{F}_{4}, \varpi_{1}\right)$. Note that $\left(\mathbf{A}_{3}, \varpi_{2}^{*}\right)=\left(\mathbf{D}_{3}, \varpi_{1}\right)$ and this good pair is included in the list in (ii).

It suffices to check that the free generators of $\mathbb{k}[R(\lambda)]^{U^{\prime}}$ given in that Table do not form a regular sequence. To this end, we point out a certain relation in $\mathbb{k}[R(\lambda)]$ using the fact the weights of generators do not form a sparse set (cf. the proof of Theorem 3.4).

The only "bad" serial case is $\left(\mathbf{A}_{r}, \varpi_{2}^{*}\right)$ with $r \geqslant 4$. The algebra $\mathbb{k}\left[\mathrm{R}\left(\varpi_{2}^{*}\right)\right]^{U}$ has free generators $f_{2 i}(1 \leqslant i \leqslant[r / 2])$ of degree $i$ and weight $\varpi_{2 i}$, and for $r$ odd, there is also the Pfaffian, which is $G$-invariant. Then $\mathbb{k}\left[\mathrm{R}\left(\varpi_{2}^{*}\right)\right]^{U^{\prime}}$ is freely generated by $f_{2}, \tilde{f}_{2}, f_{4}, \tilde{f}_{4}, \ldots$ (and the Pfaffian, if $r$ is odd). Using the 4-nodes fragments of the weight posets $\mathcal{P}\left(\varpi_{2}\right)$ and $\mathcal{P}\left(\varpi_{4}\right)$ and notation of the proof of Theorem 3.4, we construct a $U$-invariant function $f_{2} q_{4}-\tilde{f}_{2} p_{4}+p_{2} \tilde{f}_{4}-q_{2} f_{4}$ of degree 3 and weight $\varpi_{2}+\varpi_{4}-\alpha_{2}-\alpha_{3}-\alpha_{4}=\varpi_{1}+\varpi_{5}$. (Cf. Eq. (3.1).) However, there are no such nonzero $U$-invariants in $\mathbb{k}\left[R\left(\varpi_{2}^{*}\right)\right]$. This yields a relation in $\mathbb{k}\left[\mathrm{R}\left(\varpi_{2}^{*}\right)\right]$ involving free generators $f_{2}, \tilde{f}_{2}, f_{4}, \tilde{f}_{4} \in \mathbb{k}\left[\mathrm{R}\left(\varpi_{2}^{*}\right)\right]^{U^{\prime}}$.

In all other cases, we can do the same thing using a pair of generators of $\mathbb{k}[R(\lambda)]^{U}$ corresponding to suitable fundamental weights. The only difference is that one of these two $U$-invariants is not included in the minimal generating system of $\mathbb{k}[R(\lambda)]^{U^{\prime}}$ and should be expressed via some other $U^{\prime}$-invariants. Nevertheless, the resulting relation still shows that the $U^{\prime}$-invariants involved do not form a regular sequence.

For instance, consider the pair $\left(\mathbf{D}_{6}, \varpi_{6}\right)$. Here the free generators of $\mathbb{k}\left[\mathrm{R}\left(\varpi_{6}\right)\right]^{U}$ have the following degrees and weights: $\left(1, \varpi_{6}\right),\left(2, \varpi_{2}\right),\left(3, \varpi_{6}\right),\left(4, \varpi_{4}\right),(4, \underline{0})$ [3]. The invariants themselves are denoted by $f_{6}^{(1)}, f_{2}, f_{6}^{(3)}, f_{4}, F$, respectively. Starting with the $U$-invariants $f_{2}$ and $f_{4}$, we obtain, as a above, a relation of the form

$$
f_{2} q_{4}-\tilde{f}_{2} p_{4}+p_{2} \tilde{f}_{4}-q_{2} f_{4}=0
$$

in $\mathbb{k}\left[\mathrm{R}\left(\varpi_{6}^{*}\right)\right]$. However, $f_{4}$ is not a generator in $\mathbb{k}\left[\mathrm{R}\left(\varpi_{6}\right)\right]^{U^{\prime}}$. Taking the second $U^{\prime}$-invariant in each fundamental $G$-submodule, we obtain nine functions $f_{6}^{(1)}, \tilde{f}_{6}^{(1)}, f_{2}, \tilde{f}_{2}, f_{6}^{(3)}, \tilde{f}_{6}^{(3)}$, 
$f_{4}, \tilde{f}_{4}, F$ that generate $\mathbb{k}\left[\mathrm{R}\left(\varpi_{6}\right)\right]^{U^{\prime}}$. Here $f_{4}=f_{6}^{(1)} \tilde{f}_{6}^{(3)}-\tilde{f}_{6}^{(1)} f_{6}^{(3)}$ and the remaining eight functions freely generate $\mathbb{k}\left[R\left(\varpi_{6}\right)\right]^{U^{\prime}}$. Substituting this expression for $f_{4}$ in (6.1), we finally obtain the relation

$$
f_{2} q_{4}-\tilde{f}_{2} p_{4}+p_{2} \tilde{f}_{4}-q_{2}\left(f_{6}^{(1)} \tilde{f}_{6}^{(3)}-\tilde{f}_{6}^{(1)} f_{6}^{(3)}\right)=0,
$$

which shows that the free generators of $\mathbb{k}\left[R\left(\varpi_{6}\right)\right]^{U^{\prime}}$ do not form a regular sequence.

Some open problems. Let $V$ be a rational $G$-module.

$1^{\circ}$. Suppose that $V / / U$ is an affine space. Is it true that $V / / U^{\prime}$ is a complete intersection?

$2^{\circ}$. Suppose that $V / / U^{\prime}$ is an affine space and $G$ has no simple factors $S L_{2}$. Is it true that $V / / U$ is an affine space? (In [14], we have proved that $V / / G$ is an affine space, but this seems to be too modest.)

Direct computations provide an affirmative answer to both questions if $G$ is simple and $V$ is a simple $G$-module.

Acknowledgements. Part of this work was done while I was visiting MPIM (Bonn). I thank the Institute for the hospitality and inspiring environment. I am grateful to E.B. Vinberg for sending me the preprint [20].

\section{REFERENCES}

[1] А. БеРенштеЙн, А. ЗЕлЕВИнСКИЙ. Когда кратность веса равна 1? Функи. анализ и прилож., 24, № 4 (1990), 1-13 (Russian). English translation: A. BERENSTEIN and A. ZelevinSKY. When is the multiplicity of a weight equal to 1? Funct. Anal. Appl., 24 (1991), 259-269.

[2] M. Brion. Sur la théorie des invariants, Publ. Math. Univ. Pierre et Marie Curie, no. 45 (1981), pp. 1-92.

[3] M. BRION. Invariants d'un sous-groupe unipotent maximal d'un groupe semi-simple, Ann. Inst. Fourier, 33 (1983), 1-27.

[4] M. BRION. Quelques propriétés des espaces homogènes sphériques, Manuscr. Math., 55 (1986), 191198.

[5] M. BRiON, D. LunA and TH. Vust. Espaces homogènes sphériques. Invent. Math., 84 (1986), 617-632.

[6] C. Chevalley. "Fondements de la géométrie algébrique", Paris, 1958.

[7] В.И. ДАнилов. Геометрия торических многообразий, Успехи Матем. Наук, т. XXXIII, вып. 2 (1978), 85-134 (Russian). English translation: V.I. DANILOV. Geometry of toric varieties, Russian Math. Surveys, 33:2, (1978), 97-154.

[8] J.E. HumphreYs. "Reflection Groups and Coxeter Groups", Cambridge Univ. Press, 1992.

[9] H. KRAFT. "Geometrische Methoden in der Invariantentheorie", Aspekte der Mathematik D1, Braunschweig: Vieweg \& Sohn, 1984.

[10] F. GRosshans. The variety of points which are not semi-stable, Illinois J. Math. 26 (1982), 138-148.

[11] D. PAnyushev. On deformation method in Invariant Theory, Ann. Inst. Fourier, 47 (1997), 985-1012.

[12] D. PAnYUSHEv. Complexity and rank of actions in invariant theory, J. Math. Sci. (New York), 95 (1999), 1925-1985.

[13] D. PAnYUShev. Parabolic subgroups with Abelian unipotent radical as a testing site for Invariant Theory, Canad. J. Math., 51(3) (1999), 616-635. 
[14] D. PAnYushev. Actions of the derived group of a maximal unipotent subgroup on $G$-varieties, Int. Math. Res. Notices, 2010, no. 4 (2010), 674-700.

[15] В.Л. Попов. Стягивания действий редуктивных алгебраических групп, Матем. Сб., т.130 (1986), 310-334 (Russian). English translation: V.L. Popov. Contractions of actions of reductive algebraic groups, Math. USSR-Sbornik, 58 (1987), 311-335.

[16] G. SCHWARZ. Lifting smooth homotopies of orbit spaces, Publ. Math. I.H.E.S., 51 (1980), 37-135.

[17] J.-P. SERRE. “Local algebra”, Springer Monographs in Mathematics, Berlin: Springer-Verlag, 2000.

[18] Э.Б. ВиньерГ, Б.Н. КимельфЕЛЬд. Однородные области на флаговых многообразиях и сферические подгруппы полупростых групп Ли, Функи. анал. и прилож., 12, № 3 (1978), 12-19 (Russian). English translation: E.B. VinBERG and B.N. KimEL'FELD. Homogeneous domains on flag manifolds and spherical subgroups of semisimple Lie groups, Funct. Anal. Appl., 12 (1978), 168-174.

[19] Э.Б. Виньерг. Сложность действий редуктивных групп, Функи. анал. и прилож., 20, № 1 (1986), 1-13 (Russian). English translation: E.B. VINBERG. Complexity of action of reductive groups, Funct. Anal. Appl., 20 (1986), 1-11.

[20] Э.Б. ВиньеРГ, С.Г. Гиндикин. Вырождение орисфер в сферических однородных пространствах (Russian) (= Degeneration of horospheres in spherical homogeneous spaces, Preprint dated June 16, 2008; 16 pp.)

[21] Э.Б. Виньерг, А.Л. Онищик. "Семинар по группам Ли и алгебраическим группам". Москва: "Наука" 1988 (Russian). English translation: A.L. ONISHCHIK and E.B. VINBERG. "Lie groups and algebraic groups", Berlin: Springer, 1990.

[22] Э.Б. ВинБерг, В.Л. Попов. Об одном классе аффинных квазиоднородных многообразий, Изв. АН СССР. Сер. Матем., т.36 (1972), 749-764 (Russian). English translation: E.B. VINBERG and V.L. POPOV. On a class of quasihomogeneous affine varieties, Math. USSR-Izv., 6 (1972), 743-758.

[23] Э.Б. Виньерг, В.Л. Попов. "Теория Инвариантов", В кн.: Современные проблемы математики. Фундаментальные направления, т. 55, стр. 137-309. Москва: ВИНИТИ 1989 (Russian). English translation: V.L. POPOV and E.B. VInBERG. "Invariant theory", In: Algebraic Geometry IV (Encyclopaedia Math. Sci., vol. 55, pp. 123-284) Berlin Heidelberg New York: Springer 1994.

[24] D. Wehlau. Equidimensional varieties and associated cones, J. Algebra, 159 (1993), 47-53.

INSTITUTE FOR INFORMATION TRANSMISSION PROBLEMS OF THE R.A.S.,

B. KARetNyi Per. 19, Moscow 127994, Russia

E-mail address: panyushev@iitp.ru 